\title{
A Generally Weighted Moving Average Exceedance Chart
}

\author{
Niladri Chakraborty ${ }^{\mathrm{a}}$, Schalk W. Human ${ }^{\mathrm{a}}$ and Narayanaswamy Balakrishnan ${ }^{\mathrm{a}, \mathrm{b}}$ \\ ${ }^{a}$ Department of Statistics, University of Pretoria, Pretoria 0002, South Africa; \\ ${ }^{b}$ Department of Mathematics and Statistics, McMaster University, Hamilton, Ontario, \\ Canada L8S 4K1;
}

Niladri Chakraborty contact:

Email: niladriorama@gmail.com

Schalk W. Human contact:

Email: schalk.human@up.ac.za; Phone: (+27) 837591604

Narayanaswamy Balakrishnan contact:

Email: bala@mcmaster.ca; Phone: (905) 525-9140 Ext: 23420

N. Chakraborty has received his $\mathrm{PhD}$ in Mathematical Statistics from the Department of Statistics, University of Pretoria, South Africa. Presently his main area of research is statistical quality control.

S. W. Human has obtained PhD in Mathematical Statistics from the Department of Statistics, University of Pretoria, South Africa. In a worldwide collaboration, he has authored/coauthored numerous accredited peer-reviewed journal articles and has presented his research work at several national and international conferences. He also serves as a reviewer for a number of reputed international statistical journals. Based on his academic achievements, he was awarded several bursaries from University of Pretoria and the National Research Foundation, South Africa. He also has worked as a supervisor for a number of Doctoral and Master's candidates at the University of Pretoria, South Africa. His main area of research interests includes statistical quality control.

N. Balakrishnan is a distinguished university professor in the Department of Mathematics and Statistics at McMaster University, Hamilton, Ontario, Canada. Prof. Balakrishnan is also an extra-ordinary professor at the Department of Statistics, University of Pretoria. He is a fellow of the American Statistical Association, a fellow of the Institute of Mathematical Statistics, and an elected member of the International Statistical Institute. His research interests are quite wide covering a range of topics including ordered data analysis, distribution theory, quality control, reliability, survival analysis and robust inference. He is currently the editor-in-chief of Communications in Statistics and the Encyclopaedia of Statistical Sciences. 


\begin{abstract}
:
Distribution-free control charts gained momentum in recent years as they are more efficient in detecting a shift when there is a lack of information regarding the underlying process distribution. However, a distribution-free control chart for monitoring the process location often requires information on the in-control process median. This is somewhat challenging because, in practice, any information on the location parameter might not be known in advance and estimation of the parameter is therefore required. In view of this, a timeweighted control chart, labelled as the Generally Weighted Moving Average (GWMA) exceedance (EX) chart (in short GWMA-EX chart), is proposed for detection of a shift in the unknown process location; this chart is based on an exceedance statistic when there is no information available on the process distribution. An extensive performance analysis shows that the proposed GWMA-EX control chart is, in many cases, better than its contenders.
\end{abstract}

Keywords: nonparametric control chart; GWMA chart; exceedance statistic; precedence statistic; average run-length; Monte Carlo simulation.

\title{
1. Introduction
}

Control charts are efficient tools in statistical process control (SPC) that aim at efficient monitoring of streaming process and detecting changes, if any, in process performance as early as possible so that a corrective measure can be taken to ensure minimal loss due to a downfall in quality. Shewhart-type charts, proposed by Walter A. Shewhart in 1920s, might be appealing in practice for its simplicity, but time-weighted control charts such as Cumulative Sum (CUSUM) or Exponentially Weighted Moving Average (EWMA) charts have proven to be more efficient than Shewhart-type charts in detecting small persistent shift (see Montgomery [1]). Generalizing the EWMA charting procedure, Sheu and Lin [2] proposed a GWMA control chart for the normal distribution (denoted by GWMA- $\bar{X}$ chart) that has been shown to be more effective than EWMA, CUSUM and Shewhart-type charts (see Hsu et al. [3]) in detecting small shift in process. Chakraborty et al. [4] proposed a parametric GWMA chart to monitor time-between-failures when the process distribution is 
not normal. However, the underlying process distribution may not be known or satisfy the distributional assumption always. It has been observed that performance of a parametric GWMA chart deviates in departure from distributional assumption (see Chakraborty et al. [5] ) even when the process parameters are not shifted from its in-control standard. When the performance of a control chart is independent (or nearly independent) of the underlying process distribution, it is said to be a robust control chart and, for parametric GWMA chart, the in-control robustness is often adversely affected. It should be mentioned that $\mathrm{Lu} \mathrm{[6]} \mathrm{and}$ Chakraborty et al. [5] proposed GWMA control charts based on the sign statistic (denoted by GWMA-SN chart) and Wilcoxon signed-rank statistic (denoted by GWMA-SR chart), respectively, for the case when the true process median is known (Case $\mathrm{K}$ ).

In many practical situations, however, the true process median may not be known (Case U) that to some extent limits the applicability of the distribution-free GWMA charts based on sign and Wilcoxon signed-rank statistics. Exceedance (or precedence) tests are well known nonparametric two-sample tests based on the number of observations from one of the samples that exceed (or precede) a specified (say, the $r$-th) order statistic of the other sample for distributional shift. Precedence/exceedance test statistics are linearly related, and the tests based on these statistics are found to be useful in a number of applications including quality control and reliability studies with lifetime data. Balakrishnan and $\mathrm{Ng}$ [7] have provided a detail overview of the precedence/exceedance tests and their properties and applications. Balakrishnan and $\mathrm{Ng}$ [7] (see page 51) have stated that, 'Wilcoxon rank-sum test performs better than precedence tests if the underlying distribution is close to symmetry, such as the normal distribution, gamma distribution with large values of shape parameter and lognormal distribution with small values of the shape parameter. However, under some right-skewed distributions such as the exponential distribution, gamma distribution with shape parameter 2.0 and lognormal distribution with shape parameter 0.5 , the precedence tests possess higher 
power than the Wilcoxon's rank-sum test for small values of $r$. It is evident that the more right skewed the underlying distribution is, the more powerful the precedence test is.' Here, $r$ corresponds to the $r^{\text {th }}$ order statistic that is being used as a reference for the precedence chart. Chakraborti et al. [8] studied a class of nonparametric Shewhart-type charts based on precedence statistics, referred to as the Shewhart-type precedence charts. Graham et al. [9], Graham et al. [10], and Mukherjee et al. [11], respectively, studied EWMA and CUSUM charts based on exceedance statistics for small process shift. In this article, we construct a distribution-free GWMA chart based on what is known as exceedance statistic for monitoring unknown median of a streaming process. This chart is referred to as the GWMA exceedance (or GWMA-EX) chart.

In Section 2, a GWMA control chart based on exceedance statistic is designed and the necessary theoretical framework is developed. Design and implementation issues of the proposed chart are addressed in Section 3. Next, an illustrative example is provided in Section 4. Finally, some concluding remarks are made in Section 5.

\section{GWMA exceedance chart: Theoretical framework}

It is assumed that the in-control reference sample $X_{1}, X_{2}, \ldots, X_{m} \sim$ iid $F_{X}(x)$, where $F_{X}(x)$ is the cumulative distribution function (c.d.f.) of an unknown continuous distribution and $\theta \in(-\infty, \infty)$ is the unknown location parameter of interest. Now, suppose $Y_{i 1}, Y_{i 2}, \ldots, Y_{i n}$, $i=1,2,3, \ldots$, is the $i^{\text {th }}$ test sample of size $n \geq 1$, that follow an unknown continuous distribution $G_{Y}(x)=F_{X}(x-\theta)$. Note that a location model for the distribution of the test sample is assumed as we intend to design a control chart for monitoring the process location. Let $\theta_{0}$ be the unknown true value of the parameter $\theta$ and $\theta_{1}=\theta_{0}+\delta$ be the shifted parameter when the process goes out-of-control (OOC); here, $\delta$ is the location shift. The 
process is said to be in-control (IC) when $F=G$, i.e., when $\delta=0$. Let $X_{(r)}$ be the $r^{\text {th }}$ order statistic obtained from the Phase I sample of size $m$.

We define the exceedance statistic as $V_{i r}=$ the number of $Y_{i j} \geq X_{(r)}$ in the $i^{\text {th }}$ sample, for $j=1,2, \ldots, n$. For the sake of notational simplicity, we use $V_{i}$ hereafter to denote the exceedance statistic for the $i^{\text {th }}$ sample in Phase II.

\subsection{GWMA-EX plotting statistic}

GWMA-EX chart is constructed by taking a weighted average of a sequence of the $V_{i}{ }^{\prime} s$. Let $N$ denote the number of samples until the next occurrence of an event since its last occurrence. Then, by summing over all values of $N$, we can write

$$
\sum_{i=1}^{\infty} \operatorname{Pr}[N=i]=\sum_{i=1}^{t} \operatorname{Pr}[N=i]+\operatorname{Pr}[N>t]=1 .
$$

A generally weighted moving average (GWMA) is a weighted moving average (WMA) of a sequence of $V_{i}$ statistics, where the probability $\operatorname{Pr}[N=i]$ is regarded as the weight $w_{i}$ for the $i^{\text {th }}$ most recent statistic $V_{t-i+1}$ among the last $t$ of $V_{i}$ statistics. The probability $\operatorname{Pr}[N>t]$ is considered as the weight for the starting value, denoted by $Z_{0}$, which is taken as the unconditional IC expectation of $V_{i}$ given by $Z_{0}=E\left(V_{i} \mid \mathrm{IC}\right)=n\left(1-\left(\frac{r}{m+1}\right)\right)$ (see Appendix A4). Therefore, the plotting statistic for the GWMA-TBE chart is defined as

$$
Z_{t}=\sum_{i=1}^{t} \operatorname{Pr}[N=i] V_{t-i+1}+\operatorname{Pr}[N>t] Z_{0} \text { for } t=1,2, \ldots
$$

As in Sheu and Lin [2], the distribution of $N$ is taken to be $\operatorname{Pr}[N=i]=q^{(i-1)^{\alpha}}-q^{i^{\alpha}}$, where $0 \leq q<1$ and $\alpha>0$ are the two parameters; this is the discrete two-parameter Weibull distribution (see Nakagawa and Osaki [12]). So, the weights are given by $w_{i}=q^{(i-1)^{\alpha}}-q^{i^{\alpha}}$. By substituting the probability mass function (p.m.f.) of the two-parameter discrete Weibull distribution in equation (2), GWMA-EX chart plotting statistic is defined as

$$
Z_{t}=\sum_{i=1}^{t}\left(q^{(i-1)^{\alpha}}-q^{i^{\alpha}}\right) V_{t-i+1}+q^{t^{\alpha}} Z_{0}, \text { for } t=1,2, \ldots
$$


where $Z_{0}=n\left(1-\left(\frac{r}{m+1}\right)\right)$. Results A4 and A5 in Appendix show that the use of conditional expectation and variance of the sample statistic $V_{i}$ requires information about the underlying process distribution. For this reason, we determine the control limits by using unconditional IC expectation and variance of $Z_{t}$.

\subsection{Control limits}

We take $Z_{0}=n(1-a)$, where $a=\frac{r}{m+1}$. Using Result A4, the unconditional IC expectation of $Z_{t}$ can be determined as

$E\left(Z_{t}\right)=E_{X_{(r)}}\left(E\left(Z_{t} \mid X_{(r)}\right)\right)=\sum_{i=1}^{t}\left(q^{(i-1)^{\alpha}}-q^{i^{\alpha}}\right) E_{X_{(r)}}\left(E\left(V_{t-i+1} \mid X_{(r)}\right)\right)+q^{t^{\alpha}} n(1-a)$.

Thus,

$$
E\left(Z_{t}\right)=n(1-a)
$$

The unconditional IC variance of $Z_{t}$ is given by

$\operatorname{Var}\left(Z_{t}\right)=\operatorname{Var}_{X_{(r)}}\left(E\left(Z_{t} \mid X_{(r)}\right)\right)+E_{X_{(r)}}\left(\operatorname{Var}\left(Z_{t} \mid X_{(r)}\right)\right)$

Now,

$$
\begin{aligned}
& E\left(Z_{t} \mid X_{(r)}\right)=\sum_{i=1}^{t}\left(q^{(i-1)^{\alpha}}-q^{i^{\alpha}}\right) E\left(V_{t-i+1} \mid X_{(r)}\right)+q^{t^{\alpha}} n(1-a) \\
& =\left(1-q^{t^{\alpha}}\right) E\left(V_{t-i+1} \mid X_{(r)}\right)+q^{t^{\alpha}} n(1-a) .
\end{aligned}
$$

So,

$\operatorname{Var}_{X_{(r)}}\left(E\left(Z_{t} \mid X_{(r)}\right)\right)=\left(1-q^{t^{\alpha}}\right)^{2} \operatorname{Var}_{X_{(r)}}\left(E\left(V_{t-i+1} \mid X_{(r)}\right)\right)=\left(1-q^{t^{\alpha}}\right)^{2} \frac{n^{2} r(m-r+1)}{(m+1)^{2}(m+2)}$

Then,

$\operatorname{Var}\left(Z_{t} \mid X_{(r)}\right)=\sum_{i=1}^{t}\left(q^{(i-1)^{\alpha}}-q^{i^{\alpha}}\right)^{2} \operatorname{Var}\left(V_{t-i+1} \mid X_{(r)}\right)$ 
and

$E_{X_{(r)}}\left(\operatorname{Var}\left(Z_{t} \mid X_{(r)}\right)\right)=\sum_{i=1}^{t}\left(q^{(i-1)^{\alpha}}-q^{i^{\alpha}}\right)^{2} E_{X_{(r)}}\left(\operatorname{Var}\left(V_{t-i+1} \mid X_{(r)}\right)\right)=Q_{t} \frac{n r(m-r+1)}{(m+2)(m+1)}$,

where $Q_{t}=\sum_{i=1}^{t}\left(q^{(i-1)^{\alpha}}-q^{i^{\alpha}}\right)^{2}$, so that

$\operatorname{Var}\left(Z_{t}\right)=\left(1-q^{t^{\alpha}}\right)^{2} \frac{n^{2} r(m-r+1)}{(m+1)^{2}(m+2)}+Q_{t} \frac{n r(m-r+1)}{(m+1)(m+2)}=\frac{n r(m-r+1)}{(m+1)(m+2)}\left(\left(1-q^{t^{\alpha}}\right)^{2} \frac{n}{m+1}+Q_{t}\right)$.

Thus, the unconditional IC variance of $Z_{t}$ is

$$
\begin{gathered}
\operatorname{Var}\left(Z_{t}\right)=\frac{n r(m-r+1)}{(m+1)(m+2)}\left(\left(1-q^{t^{\alpha}}\right)^{2} \frac{n}{m+1}+Q_{t}\right) \\
\quad=\frac{n a(1-a)}{m+2}\left(\left(1-q^{t^{\alpha}}\right)^{2} n+Q_{t}(m+1)\right),
\end{gathered}
$$

where $Q_{t}=\sum_{i=1}^{t}\left(q^{(i-1)^{\alpha}}-q^{i^{\alpha}}\right)^{2}$

So, the exact control limits are given by

$$
U C L_{e} / L C L_{e}=n(1-a) \pm L \sqrt{\frac{n a(1-a)}{m+2}\left(\left(1-q^{t^{\alpha}}\right)^{2} n+Q_{t}(m+1)\right)}
$$

Thus, the steady-state control limits are given by

$$
U C L_{s} / L C L_{s}=n(1-a) \pm L \sqrt{\frac{n a(1-a)}{m+2}(n+Q(m+1))}
$$

with centre-line being $C L=n(1-a)$,

where $Q=\lim _{t \rightarrow \infty} Q_{t}=\lim _{t \rightarrow \infty} \sum_{i=1}^{t}\left(q^{(i-1)^{\alpha}}-q^{i^{\alpha}}\right)^{2}$ and $a=\frac{r}{m+1}$.

The subscripts ' $e$ ' and ' $s$ ' refer to the exact and steady-state control limits, respectively. 
The following points are now worth noting:

- Steady-state control limits are used in order to simplify the application/implementation of the chart. For the sake of notational simplicity, we use $U C L, L C L$ hereafter to denote the steady-state control limits;

-We study two-sided GWMA-EX charts with symmetrically placed control limits, i.e., equidistant from the centreline $C L$. The methodology can be easily modified wherein an one-sided chart is more meaningful or when a two-sided control chart with asymmetric control limits is necessary;

- If any charting statistic $Z_{t}$ plots on or outside either of the control limits given in Eq. (7), a signal is given and the process is declared to be OOC. Otherwise, the process is said to be IC, which implies that no location shift has occurred, and so the charting procedure continues.

In the next section, we discuss the design and implementation of the proposed GWMA-EX chart in more detail.

\section{The design and implementation of GWMA-EX chart}

Run-length distribution and its characteristics are commonly used measures to design and study the performance of a control chart. The average run-length $(A R L)$ is a popular measure for a chart's performance. The design of a control chart typically involves the calculation of the chart parameters for a pre-specified IC $A R L$ (denoted by $A R L_{0}^{*}$ ). Computational aspects of the run-length distribution for GWMA-EX chart are described next.

\subsection{Computation of the run-length distribution}

Computation of the run-length distribution for GWMA-EX chart requires some effort. This is because of the involvement of $X_{(r)}$, which is the $r^{\text {th }}$ order statistic from the reference sample, is itself a random variable. There are a number of methods available to calculate the run- 
length distribution of a time-weighted control chart. Three methods are discussed below in the context of the proposed chart along with their pros and cons.

\subsubsection{Exact approach}

Suppose the run-length random variable is denoted by $R$, and $A_{i}$ denotes the signalling event at the $i^{t h}$ sample. For any $i \geq 1$, the complimentary event $A_{i}^{c}$ can be written as $A_{i}^{c}=[L C L<$ $\left.Z_{i}<U C L\right] \equiv\left[L_{i}<V_{i}<U_{i}\right]$, where, for $i=2,3, \ldots$,

$$
\left\{\begin{array}{l}
U_{i}=\frac{U C L-\sum_{j=2}^{i}\left(q^{(j-1)^{\alpha}}-q^{j^{\alpha}}\right) V_{i-j+1}-q^{i^{\alpha}} n(1-a)}{1-q}, \\
L_{i}=\frac{L C L-\sum_{j=2}^{i}\left(q^{(j-1)^{\alpha}}-q^{j^{\alpha}}\right) V_{i-j+1}-q^{i^{\alpha}} n(1-a)}{1-q},
\end{array}\right.
$$

and $U_{1}=\frac{U C L-q n(1-a)}{1-q}, L_{1}=\frac{L C L-q n(1-a)}{1-q}$ and $a=\frac{r}{m+1}$. The conditional $A R L$ is given by $A R L \mid X_{(r)}=\sum_{r=0}^{\infty} I_{\mathrm{r}}$, where $I_{r}=\sum_{L_{1}}^{U_{1}} \sum_{L_{2}}^{U_{2}} \ldots \sum_{L_{r}}^{U_{r}}\left(\prod_{i=1}^{r} \operatorname{Pr}\left[V_{i}=v_{i} \mid X_{(r)}\right]\right)$ for $r=1,2,3, \ldots$ and $I_{0}=1$ (see Appendix A6). $\operatorname{Pr}\left[V_{i}=v_{i} \mid X_{(r)}\right]$ is given in Appendix A1. Unconditioning the conditional $A R L$ given in Appendix A6, the unconditional $A R L$ is, therefore,

$$
A R L=E_{X_{(r)}}\left(A R L \mid X_{(r)}\right)=1+\sum_{r=1}^{\infty} E_{X_{(r)}}\left(I_{r}\right)
$$

Obtaining $E_{X_{(r)}}\left(I_{r}\right)$, then replacing $\operatorname{Pr}\left[V_{i}=v_{i}\right]$ (see Appendix A2) in (9), one can obtain a closed-form expression for the unconditional $A R L$ as

$$
A R L=1+\sum_{r=1}^{\infty} \sum_{L_{1}}^{U_{1}} \sum_{L_{2}}^{U_{2}} \ldots \sum_{L_{r}}^{U_{r}}\left(\prod_{i=1}^{r}\left(\frac{\left(\begin{array}{c}
v_{i}+m-r \\
v_{i}
\end{array}\right)\left(\begin{array}{c}
n-v_{i}+r-1 \\
n-v_{i}
\end{array}\right)}{\left(\begin{array}{c}
m+n \\
n
\end{array}\right)}\right)\right)
$$

Thus, a control chart based on the exceedance statistic $V_{i}$ is distribution-free when the process is IC and this is referred to as 'IC robustness'. This is a very important property of a control chart as variation in process performance is not desired for a change in the underlying process 
distribution when the process is IC. However, $A R L$ value computation by (10) is quite involved even with the use of a computer.

\subsubsection{Markov chain approach}

Markov chain approach is another familiar approach to obtain the run-length distribution and properties of a time-weighted control chart. However, Sheu and Lin [2], Sheu and Yang [13], Lu [6] and Chakraborty et al. [4] have mentioned that $A R L$ for the GWMA charts cannot be obtained very easily by Markov chain approach. In a Case U GWMA chart, computation of unconditional $A R L$ is even heftier with the Markov chain approach.

\subsubsection{Monte Carlo simulation approach}

Monte Carlo simulation approach is, therefore, used to approximate the $A R L$ values. To estimate the unconditional run-length distribution and its characteristics through Monte Carlo simulation approach for GWMA-EX chart, the following steps are to be adopted:

1) In order to simulate the run-length distribution, one needs to specify a process distribution $F_{X}(x)$, the IC distribution parameter $\theta_{0}$, reference and test sample sizes $m$ and $n$, the shift to be detected denoted by $\delta$, and the chart parameters $(q, \alpha, L)$;

2) Generate a reference sample from the specified process distribution $F_{X}(x)$ and obtain $X_{(r)}$

3) Generate a test sample of size $n$ and calculate $V_{i}$ by counting the number of $Y$ observations in the $i^{\text {th }}$ test sample that are at least as large as $X_{(r)}$. The test sample should be drawn from $F_{X}(x-\theta)$, where $\theta=\theta_{0}$ when simulating an IC run-length distribution and $\theta=\theta_{0}+\delta$ when simulating an OOC run-length distribution;

4) Calculate $Q$ and obtain the steady-state control limits in Eq. (7) by using the ( $q, \alpha, L)$ values chosen in step (1); 
5) Take $Z_{0}=n\left(1-\left(\frac{r}{m+1}\right)\right)$ and calculate the plotting statistic $Z_{t}$ given in Eq. (3) for $t=1,2, \ldots$ and compare each $Z_{t}$ with the steady-state control limits obtained;

6) The number of test samples until a $Z_{t}$ falls on or outside either of the limits is taken as an observation from the run-length distribution;

7) Repeat steps (1)-(6) a large number of times, say, 10,000 times, that gives us 10,000 observations from the run-length distribution. Using this set of run-length observations, we can obtain different distributional properties such as $A R L, S D R L$ or different percentile of the run-length distribution;

\subsection{The in-control design and implementation}

For chosen values of $m$ and $n$, the two parameters $q$ and $\alpha$ are varied over a certain range of values and for each $(q, \alpha)$ combination, the values of the charting constant, i.e., $L>0$, are obtained so that the attained IC $A R L$ (denoted by $A R L_{0}$ ) is close to (in this case, slightly above or below for the use of simulation) the nominal or specified value $A R L_{0}^{*}$. The typical industry standards for $A R L_{0}^{*}$ are 370 or 500 and we consider the former in our study. The typical recommendation for the smoothing parameter $0<\lambda \leq 1$ for an EWMA chart is to choose smaller values for smaller shifts (see Montgomery [1], page 423). Because the GWMA chart reduces to an EWMA chart when $q=1-\lambda$ and $\alpha=1$, a larger value of $q$, i.e., closer to 1 , should be a reasonable choice for the GWMA chart to detect small shifts. To this end, Sheu and Lin [2] noted that $(q, \alpha)$ combinations in the intervals $0.5 \leq q \leq 0.9$ and $0.5 \leq \alpha<1$ enhanced the sensitivity of the GWMA- $\bar{X}$ chart and outperformed the EWMA- $\bar{X}$ chart for small shifts (i.e., less than 1.5 standard deviations in the location). In our study, we set $m=49,99, n=5,10$, and then considered the range $q=0.8,0.9,0.95$ and $\alpha=0.7,0.8$, $0.9,1.0,1.3$, respectively. By using simulations along with a grid search algorithm, we obtained the charting constant $L>0$ for the chosen $(q, \alpha)$ combination and specified values 
Table 1: IC and OOC $A R L$ for different combinations of $(q, \alpha, L)$ for different shift $\delta$ when $m=49, n=$ 5, 10 with $A R L_{0}^{*}=370$.

\begin{tabular}{|c|c|c|c|c|c|c|c|c|c|c|c|}
\hline & & & & \multicolumn{8}{|c|}{$\delta$} \\
\hline$m=49$ & $q$ & $\alpha$ & $L$ & 0 & 0.05 & 0.1 & 0.25 & 0.5 & 0.75 & 1.0 & 1.5 \\
\hline \multirow[t]{15}{*}{$n=5$} & 0.8 & 0.7 & 2.033 & 369.48 & 358.96 & 323.66 & 182.36 & 31.79 & 10.41 & 6.51 & 4.14 \\
\hline & & 0.8 & 2.112 & 369.16 & 360.33 & 329.31 & 185.74 & 31.77 & 10.12 & 6.26 & 4.06 \\
\hline & & 0.9 & 2.184 & 370.09 & 360.94 & 332.66 & 185.72 & 32.22 & 9.90 & 6.06 & 4.03 \\
\hline & & 1.0 & 2.248 & 370.13 & 363.06 & 330.50 & 187.49 & 32.94 & 9.79 & 5.95 & 3.92 \\
\hline & & 1.3 & 2.382 & 371.26 & 355.39 & 327.53 & 195.61 & 39.49 & 10.37 & 5.82 & 3.84 \\
\hline & 0.9 & 0.7 & 1.464 & 372.82 & 352.92 & 323.44 & 171.05 & 31.70 & 11.22 & 7.68 & 5.27 \\
\hline & & 0.8 & 1.595 & 371.98 & 358.17 & 338.32 & 172.02 & 29.60 & 10.84 & 7.37 & 5.18 \\
\hline & & 0.9 & 1.713 & 370.14 & 364.75 & 332.21 & 179.75 & 30.23 & 10.41 & 7.08 & 5.07 \\
\hline & & 1.0 & 1.819 & 368.93 & 355.72 & 336.79 & 180.44 & 29.36 & 10.10 & 6.79 & 4.93 \\
\hline & & 1.3 & 2.069 & 369.11 & 365.8 & 329.52 & 185.43 & 33.33 & 9.52 & 6.22 & 4.52 \\
\hline & 0.95 & 0.7 & 0.951 & 372.98 & 353.57 & 334.12 & 166.62 & 31.19 & 13.22 & 9.13 & 6.43 \\
\hline & & 0.8 & 1.089 & 370.34 & 347.62 & 310.26 & 169.06 & 30.00 & 12.65 & 8.88 & 6.38 \\
\hline & & 0.9 & 1.228 & 368.56 & 349.78 & 321.38 & 165.64 & 30.6 & 12.08 & 8.6 & 6.3 \\
\hline & & 1.0 & 1.363 & 373.83 & 359.62 & 328.24 & 174.2 & 30.64 & 11.48 & 8.26 & 6.2 \\
\hline & & 1.3 & 1.715 & 368.95 & 358.95 & 328.1 & 182.08 & 29.6 & 10.17 & 7.29 & 5.63 \\
\hline \multirow[t]{15}{*}{$n=10$} & 0.8 & 0.7 & 1.716 & 371.34 & 346.20 & 321.47 & 163.70 & 21.44 & 6.64 & 4.09 & 2.71 \\
\hline & & 0.8 & 1.799 & 367.01 & 369.04 & 332.38 & 170.45 & 21.60 & 6.22 & 4.08 & 2.86 \\
\hline & & 0.9 & 1.871 & 370.80 & 358.76 & 331.82 & 169.95 & 21.93 & 6.06 & 3.97 & 2.83 \\
\hline & & 1.0 & 1.943 & 368.99 & 353.45 & 328.11 & 175.56 & 23.78 & 6.07 & 3.89 & 2.81 \\
\hline & & 1.3 & 2.119 & 372.48 & 373.53 & 335.21 & 185.60 & 26.17 & 5.84 & 3.68 & 2.77 \\
\hline & 0.9 & 0.7 & 1.164 & 372.88 & 353.62 & 319.78 & 161.54 & 22.83 & 7.49 & 5.05 & 3.50 \\
\hline & & 0.8 & 1.274 & 374.14 & 357.50 & 321.47 & 167.35 & 21.34 & 7.30 & 4.98 & 3.56 \\
\hline & & 0.9 & 1.380 & 374.68 & 356.03 & 320.98 & 171.01 & 22.56 & 6.87 & 4.80 & 3.49 \\
\hline & & 1.0 & 1.479 & 370.06 & 359.48 & 324.26 & 166.10 & 19.40 & 6.88 & 4.66 & 3.42 \\
\hline & & 1.3 & 1.751 & 372.46 & 371.39 & 328.54 & 173.18 & 21.61 & 6.08 & 4.40 & 3.36 \\
\hline & 0.95 & 0.7 & 0.738 & 370.05 & 350.26 & 323.10 & 155.84 & 26.30 & 9.15 & 6.24 & 4.42 \\
\hline & & 0.8 & $\mathbf{0 , 8 5 4}$ & 370.13 & 344.66 & 326.17 & 160.90 & 22.74 & 8.82 & 6.29 & 4.53 \\
\hline & & 0.9 & 0,967 & 369.04 & 361.43 & 329.72 & 169.01 & 22.60 & 8.44 & 6.19 & 4.57 \\
\hline & & 1.0 & 1,079 & 369.79 & 361.06 & 326.51 & 158.52 & 24.04 & 8.05 & 5.99 & 4.56 \\
\hline & & 1.3 & 1,396 & 372.89 & 347.82 & 328.52 & 175.51 & 21.78 & 7.18 & 5.47 & 4.32 \\
\hline
\end{tabular}


Table 2: IC and OOC $A R L$ for different combinations of $(q, \alpha, L)$ for different shift $\delta$ when $m=99, n=$ 5, 10 with $A R L_{0}^{*}=370$.

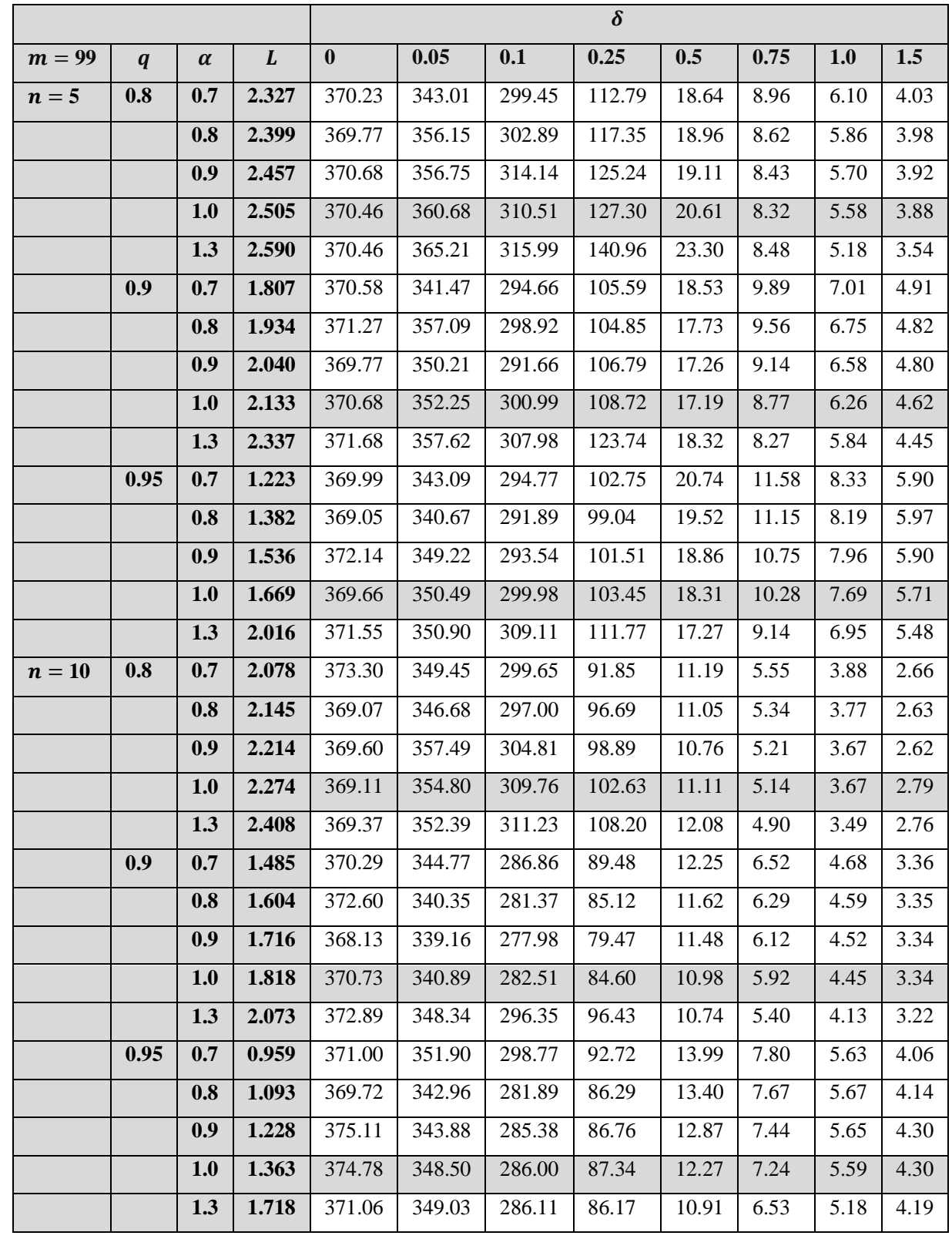

of $m$ and $n$, so that the attained $A R L_{0}$ is approximately equal to $A R L_{0}^{*}=370$. The values of $L$ for which the attained $A R L_{0}$ values are close to 370 are reported in Tables 1 and 2 along with the corresponding $A R L_{0}$ values in the column for $\delta=0$. We compare our simulation results with Graham et al. [9] to ensure that the results are consistent in two different studies. For instance, when $m=99, n=5$, we have $(q=0.9, \alpha=1, L=2.133)$ wherein Graham et al. [9], the parameters are $(\lambda=0.1, L=2.132)$ for $A R L_{0} \approx A R L_{0}^{*}$, since $q=1-\lambda$. The $L$ 
values in Tables 1 and 2 are useful in the design and implementation of the GWMA-EX chart.

It is worth noting that, as a usual practice, we focus mostly on the median of the reference sample for $X_{(r)}$, i.e., the $r^{\text {th }}$ order statistic is the median from Phase I sample as described in Section 2. However, we conducted a short performance study for the proposed GWMA-EX chart with $25^{\text {th }}$ and $75^{\text {th }}$ percentiles of the Phase I sample taken as $X_{(r)}$. We observed certain issues arising while using other percentiles for GWMA-EX chart instead of the median of the Phase I sample in performing process monitoring when the direction of the shift to be detected is not specified and for this reason we recommend the use of median of the Phase I sample for the GWMA-EX chart. This is discussed in detail in the next section. Although the choice of reference sample order statistic and overcoming the challenges arising thereafter are interesting problems to study on their own merit, in this paper, we limit ourselves mostly to the choice of median since it is a robust measure of the central tendency of distributions of any shape and a popular choice of percentiles in practice. We also avoid the complications arising from the use of other percentiles by using median of the Phase I reference sample.

Fig. 1 shows boxplot-like graphs for IC run-length distribution of GWMA-EX charts for different $(q, \alpha, L)$ combinations when $m=49$ and $n=5$. It can be observed that the runlength distribution is a heavily right-skewed distribution. The design of the GWMA-EX chart is described in this section in order to provide a general guideline to practitioners. Considering practical limitations, we only select a few parameter combinations, as stated above, but this study can easily be extended for a larger range of parameter values following the same guidelines as we have provided here. In the next section, we discuss the OOC performance of the proposed GWMA-EX chart for different shift sizes. 
Figure 1. Boxplot-like graphs for IC run-length distribution of GWMA-EX charts for different $(q, \alpha, L)$ combinations when $m=49, n=5$.

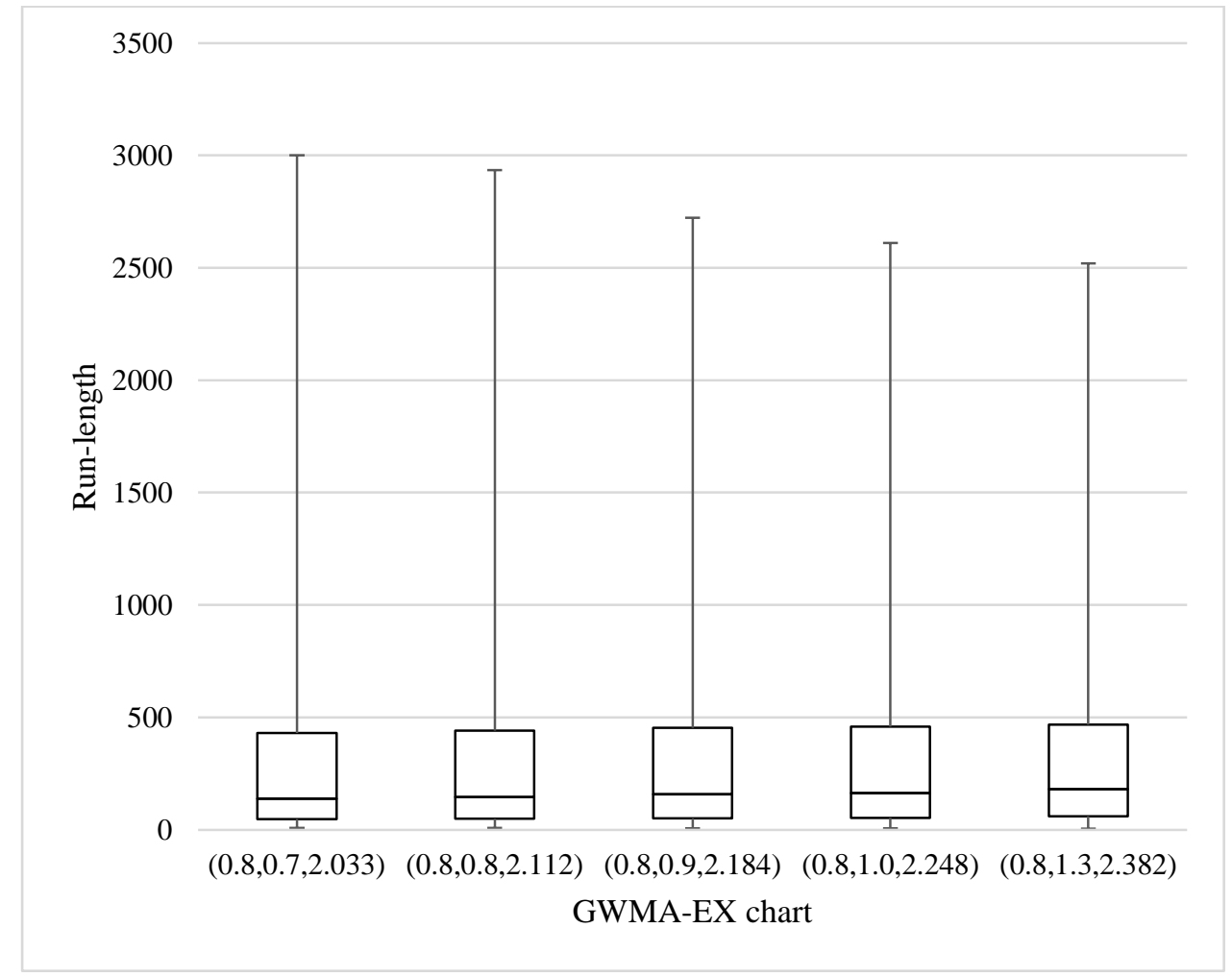

\subsection{The out-of-control performance}

The GWMA charts are generally more sensitive than the EWMA charts in detecting small shifts (see the results in Sheu and Lin [2], Lu [6] and Sheu and Yang [13]). As observed by Graham et al. [9], EWMA exceedance chart (EWMA-EX chart) can be taken as a competitive alternative to many parametric and nonparametric control charts (see Graham et al. [9] for more details). It is reasonable now to compare the proposed GWMA-EX chart with the GWMA- $\bar{X}$ chart and the EWMA-EX chart for normal and a number of non-normal underlying process distributions.

To study the performance of the GWMA-EX chart, we use the combinations of the parameters from Table 1; these combinations ensure that the attained $A R L_{0}$ is close to 370 . In order to perform a comparison study between different control charts (or between different choices of chart parameters), it is important to ensure the IC performance is similar for all 
competing charts. Once different competing charts are placed at similar IC levels, one can select a control chart with minimal $A R L_{1}$ performance as a good chart for practical implementation.

From Tables 1 and 2, we note that in general, multiple combinations of the parameters $(q, \alpha, L)$ will yield the same $A R L_{0}$ for some chosen or specified values of $m$ and $n$. This is somewhat challenging because, apart from desiring a sufficiently large $A R L_{0}, A R L_{1}$ should be small for an effective GWMA-EX chart. Therefore, the $(q, \alpha, L)$ combination with the minimum $A R L_{1}$ obtained in the scope of our simulation study for a specified shift $\delta$ is said to be the relative optimal combination. The relative optimal design of the GWMA-EX chart consists of specifying the desired $A R L_{0}$ and $A R L_{1}$ values as well as the magnitude of the process shift that is anticipated and then select that $(q, \alpha, L)$ combination that provides the desired $A R L$ performance; typically, the $(q, \alpha, L)$ combination with the minimum $A R L_{1}$ is selected. For example, in Table 1 , the combination $(q=0.95, \alpha=0.8, L=1.089)$ has the minimum $A R L_{1}=347.62$ among the chosen range of parameters for shift $\delta=0.05$ for $m=49$ and $n=5$. A detailed study on the optimal design of the GWMA-EX chart is not within the scope of this article. We study the $A R L_{1}$ performance of the proposed GWMA-EX chart with the specified range of parameter combinations for shift $\delta=0.05,0.10,0.25,0.50$, $0.75,1.0$ and 1.5 . Because the IC distribution of the exceedance statistic is symmetric for $X_{(r)}$ taken as median, to study $A R L_{1}$ performance, only the positive shifts (i.e., increases in the mean/median) are considered; the results are equally applicable for negative or downward shifts. Since the Shewhart-type charts are traditionally known to be efficient in detecting large shifts, we do not consider shifts more than 1.5; the focus of this study is on efficiently detecting small to moderate shifts. 
Tables 1 and 2 show the IC and OOC ARL performance of the proposed GWMA-EX chart with different combinations of $(q, \alpha, L)$ when shift $\delta=0.05,0.10,0.25,0.50,0.75,1.0$ and 1.5 for $m=49,99$ and $n=5,10$. As observed from the $A R L$ performance,

- GWMA-EX chart, with a suitably chosen $\alpha<1$, performs generally better than the EWMA-EX chart (i.e., when $\alpha=1$ ) for $\delta \leq 0.5$. For example, in order to detect a shift of $\delta=0.25$, a GWMA-EX chart with $(q=0.8, \alpha=0.7, L=2.033)$ has $A R L_{1}=$ 182.36 whereas the EWMA-EX chart with $(q=0.8, \alpha=1.0, L=2.248)$ has $A R L_{1}=$ 187.49 when $m=49$ and $n=5$. However, many cases such as a GWMA-EX chart with $q=0.9, \alpha=0.7,0.8,0.9$ are worse than the EWMA-EX chart at $\alpha=1$ when $m=$ 49 and $n=5$;

- For moderate shift of $\delta>0.5$, the GWMA-EX chart with $\alpha>1$ works in generally better than the EWMA-EX chart, especially at larger design parameter $q$.

Although $A R L$ and $S D R L$ are popular performance measures, we also look at a number of percentiles including $1^{\text {st }}, 5^{\text {th }}, 25^{\text {th }}, 50^{\text {th }}$ (or $\left.M R L\right), 75^{\text {th }}, 95^{\text {th }}$ and $99^{\text {th }}$ percentiles of the runlength distribution for the GWMA-EX chart. Results are available upon request from the authors.

We also ran a comparative performance study for the GWMA-EX chart when the $r^{\text {th }}$ order statistic from Phase I sample, i.e., $X_{(r)}$ is taken as the $75^{\text {th }}$ and $25^{\text {th }}$ percentiles instead of the median. The corresponding results are given in Table 3. Our study shows that for an upward shift, the choice of $X_{(r)}=75^{\text {th }}$ percentile of the Phase I sample works significantly better than the median irrespective of the choice of $(q, \alpha, L)$ or the choice of shift $\delta$. On the other hand, for $X_{(r)}=25^{\text {th }}$ percentile, the run-length distribution of the GWMA-EX chart often encounters bias, i.e., $A R L_{1}$ is often more than $A R L_{0}$ and the performance is uniformly worse than when $X_{(r)}$ is taken as the median of the Phase I sample. For the sake of brevity, a 
selection of results is presented in Table 3. A more detailed performance study is carried out and results are available upon request from the authors.

For the ease of performance comparison based on $A R L$ profiles of two charts, Fig. 2 is provided below for specific choice of $q, \alpha$ and respective $L$. It is clear that, for an upward shift in the process, $X_{(r)}=75^{\text {th }}$ percentile has uniformly better performance than $X_{(r)}=$ median, but $X_{(r)}=25^{\text {th }}$ percentile has uniformly lower performance than $X_{(r)}=$ median. One can similarly conduct a study for downward shift in process as well in which we would expect $X_{(r)}=25^{\text {th }}$ percentile to have uniformly better performance.

Figure 2. IC and OOC ARL for $X_{(r)}=75$ th, 50th and 25th percentiles of the Phase I sample for upward shift when $q=0.8, \alpha=0.9$.

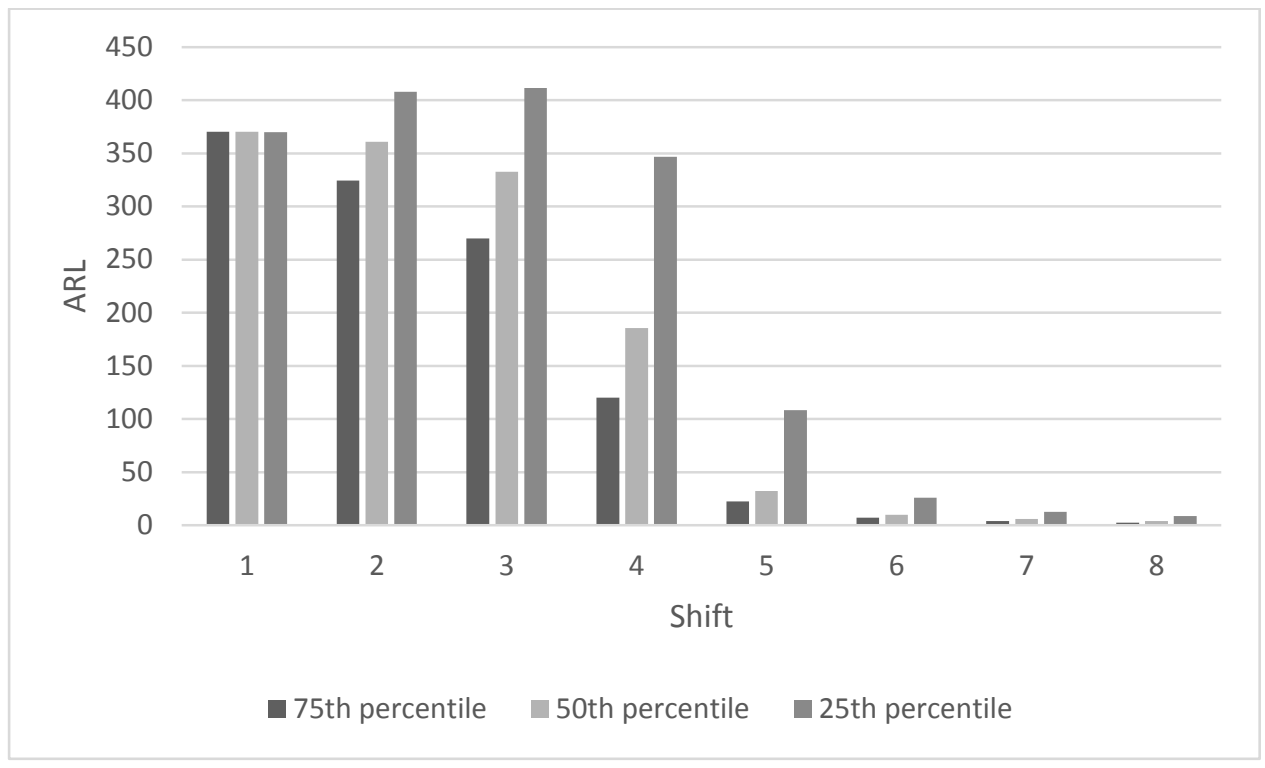


Table 3: $A R L$ values for $X_{(r)}=75^{\text {th }}, 50^{\text {th }}$ and $25^{\text {th }}$ percentiles of the Phase I sample for different shift $\delta$ when $m=49, n=5$.

\begin{tabular}{|c|c|c|c|c|c|c|c|c|c|c|c|}
\hline \multirow[b]{2}{*}{$X_{(r)}$} & \multirow[b]{2}{*}{$q$} & \multirow[b]{2}{*}{$\alpha$} & \multicolumn{9}{|c|}{$\delta$} \\
\hline & & & $L$ & 0 & 0.05 & 0.1 & 0.25 & 0.5 & 0.75 & 1 & 1.5 \\
\hline $75^{\text {th }}$ percentile & 0.8 & 0.9 & 2.120 & 370.18 & 324.31 & 269.85 & 120.28 & 22.42 & 7.07 & 4.12 & 2.54 \\
\hline $50^{\text {th }}$ percentile & & & 2.184 & 370.09 & 360.94 & 332.66 & 185.72 & 32.22 & 9.90 & 6.06 & 4.03 \\
\hline $25^{\text {th }}$ percentile & & & 2.123 & 369.86 & 408.11 & 411.32 & 346.7 & 108.23 & 25.89 & 12.85 & 8.92 \\
\hline $75^{\text {th }}$ percentile & & 1.0 & 2.174 & 371.02 & 325.56 & 265.33 & 122.28 & 21.78 & 7.12 & 4.07 & 2.51 \\
\hline $50^{\text {th }}$ percentile & & & 2.248 & 370.13 & 363.06 & 330.50 & 187.49 & 32.94 & 9.79 & 5.95 & 3.92 \\
\hline $25^{\text {th }}$ percentile & & & 2.175 & 369.82 & 403.41 & 422.48 & 354.66 & 116.03 & 25.30 & 12.59 & 8.1 \\
\hline $75^{\text {th }}$ percentile & & 1.3 & 2.289 & 369.11 & 321.64 & 262.60 & 121.94 & 21.97 & 6.94 & 3.98 & 2.44 \\
\hline $50^{\text {th }}$ percentile & & & 2.382 & 371.26 & 355.39 & 327.53 & 195.61 & 39.49 & 10.37 & 5.82 & 3.84 \\
\hline $25^{\text {th }}$ percentile & & & 2.291 & 369.08 & 412.64 & 440.00 & 410.45 & 152.92 & 35.41 & 14.69 & 7.59 \\
\hline $75^{\text {th }}$ percentile & 0.9 & 0.9 & 1.695 & 370.27 & 338.72 & 303.24 & 145.41 & 26.57 & 8.39 & 5.21 & 3.34 \\
\hline $50^{\text {th }}$ percentile & & & 1.713 & 370.14 & 364.75 & 332.21 & 179.75 & 30.23 & 10.41 & 7.08 & 5.07 \\
\hline $25^{\text {th }}$ percentile & & & 1.694 & 370.33 & 383.32 & 384.48 & 271.68 & 63.41 & 18.83 & 12.18 & 9.09 \\
\hline $75^{\text {th }}$ percentile & & 1.0 & 1.795 & 369.40 & 331.99 & 296.41 & 147.05 & 28.43 & 8.20 & 5.08 & 3.34 \\
\hline $50^{\text {th }}$ percentile & & & 1.819 & 368.93 & 355.72 & 336.79 & 180.44 & 29.36 & 10.10 & 6.79 & 4.93 \\
\hline $25^{\text {th }}$ percentile & & & 1.794 & 370.73 & 383.24 & 379.97 & 273.48 & 66.44 & 18.08 & 11.67 & 8.82 \\
\hline $75^{\text {th }}$ percentile & & 1.3 & 2.036 & 371.98 & 333.27 & 294.29 & 145.21 & 28.24 & 8.05 & 4.72 & 3.17 \\
\hline $50^{\text {th }}$ percentile & & & 2.069 & 369.11 & 365.80 & 329.52 & 185.43 & 33.33 & 9.52 & 6.22 & 4.52 \\
\hline $25^{\text {th }}$ percentile & & & 2.032 & 374.05 & 383.29 & 392.55 & 302.79 & 82.67 & 20.48 & 10.96 & 7.77 \\
\hline $75^{\text {th }}$ percentile & 0.95 & 0.9 & 1.227 & 371.01 & 347.58 & 304.70 & 155.50 & 32.10 & 10.11 & 6.52 & 4.21 \\
\hline $50^{\text {th }}$ percentile & & & 1.228 & 368.56 & 349.78 & 321.38 & 165.64 & 30.60 & 12.08 & 8.60 & 6.30 \\
\hline $25^{\text {th }}$ percentile & & & 1.230 & 368.20 & 376.61 & 358.33 & 235.69 & 56.76 & 19.90 & 14.07 & 11.04 \\
\hline $75^{\text {th }}$ percentile & & 1.0 & 1.365 & 371.00 & 339.84 & 310.56 & 156.06 & 31.86 & 9.85 & 6.42 & 4.25 \\
\hline $50^{\text {th }}$ percentile & & & 1.363 & 373.83 & 359.62 & 328.24 & 174.20 & 30.64 & 11.48 & 8.26 & 6.20 \\
\hline $25^{\text {th }}$ percentile & & & 1.359 & 368.73 & 373.37 & 362.65 & 236.59 & 54.07 & 18.48 & 13.02 & 10.12 \\
\hline $75^{\text {th }}$ percentile & & 1.3 & 1.704 & 368.09 & 344.24 & 302.71 & 156.53 & 29.47 & 8.98 & 5.85 & 4.10 \\
\hline $50^{\text {th }}$ percentile & & & 1.715 & 368.95 & 358.95 & 328.10 & 182.08 & 29.60 & 10.17 & 7.29 & 5.63 \\
\hline $25^{\text {th }}$ percentile & & & 1.704 & 371.06 & 377.53 & 369.95 & 259.94 & 63.10 & 17.31 & 11.23 & 8.76 \\
\hline
\end{tabular}

A performance study for the GWMA-EX chart based on the median run-length $(M R L)$ is being performed for different $X_{(r)}$. Sample size is taken as $m=100, n=5$ and a nominal $M R L$ (denoted by $M R L^{*}$ ) is taken as $M R L^{*}=350$ as a standard selection. For given $m, n$ and 
$(q, \alpha)$, we obtain $L$ values so that the attained $M R L$ is close to the nominal $M R L^{*}=350$ when $X_{(r)}$ is taken as $75^{\text {th }}, 50^{\text {th }}$ and $25^{\text {th }}$ percentiles. These are reported in Table 4 , and they show a similar result as in $A R L$-based study. The problem of bias in the run-length distribution still persists with $X_{(r)}=25^{\text {th }}$ percentile and it has uniformly poorer performance than $X_{(r)}=$ median for upward shift. This is displayed in Fig. 3. Therefore, there is no significant improvement observed in $M R L$-based performance study.

According to the study, it is not recommended for a practitioner to use a percentile other than median whenever the direction of shift is not specified to be detected. However, given the direction of the shift, upward or downward, it may be better to use accordingly an upper or lower percentile to obtain a faster shift detection. A similar study based on $M R L$ can be performed for downward shift as well.

Figure 3. IC and OOC MRL for $X_{(r)}=75$ th, 50th and 25th percentiles of the Phase I sample for upward shift when $q=0.8, \alpha=0.9$.

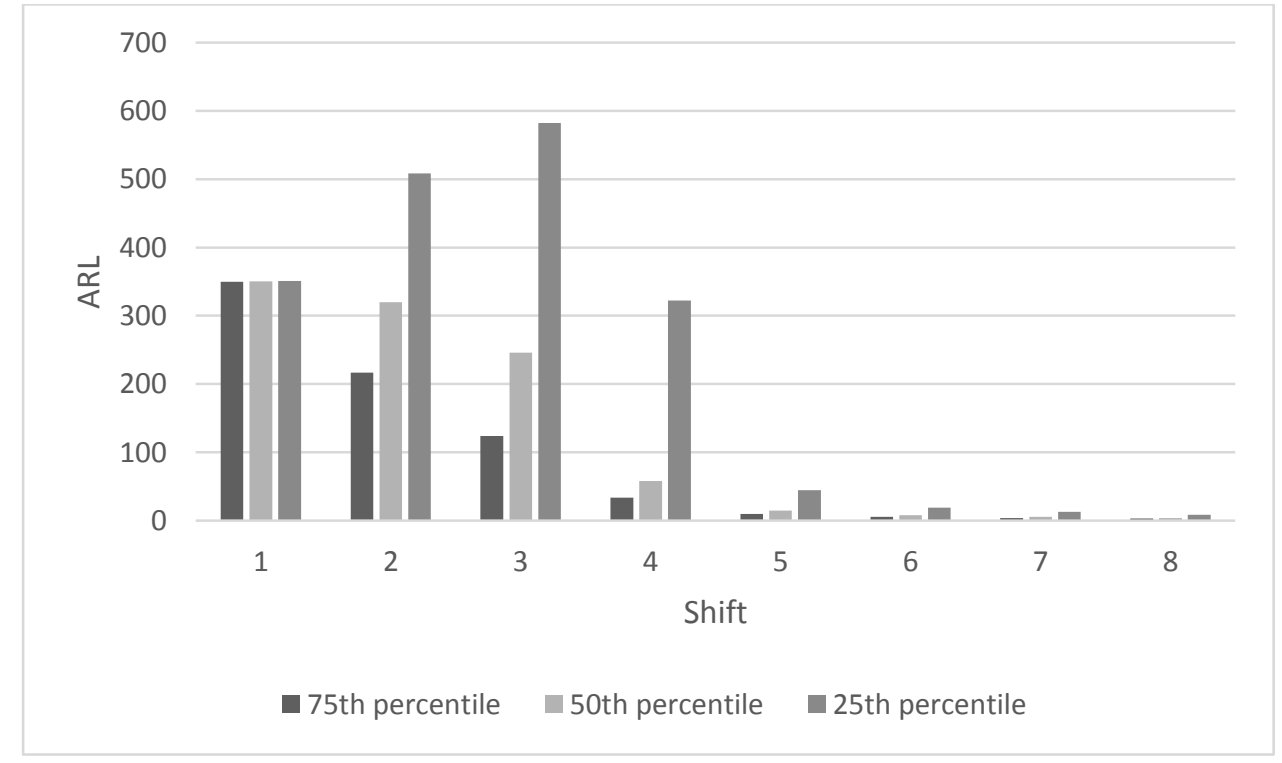


Table 4: MRL values for $X_{(r)}=7^{\text {th }}$ and $50^{\text {th }}$ percentiles of the Phase I sample for different shift $\delta$ when $m=100, n=5$.

\begin{tabular}{|c|c|c|c|c|c|c|c|c|c|c|c|}
\hline \multirow[b]{2}{*}{$\boldsymbol{X}_{(r)}$} & \multirow[b]{2}{*}{$q$} & \multirow[b]{2}{*}{$\alpha$} & \multicolumn{9}{|c|}{$\delta$} \\
\hline & & & $L$ & 0 & 0.05 & 0.1 & 0.25 & 0.5 & 0.75 & 1 & 1.5 \\
\hline $75^{\text {th }}$ percentile & 0.8 & 0.9 & 2.596 & 350.00 & 217.00 & 124.00 & 34.00 & 10.00 & 6.00 & 4.00 & 3.00 \\
\hline $50^{\text {th }}$ percentile & & & 2.620 & 350.5 & 320.00 & 246.00 & 58.00 & 15.00 & 8.00 & 6.00 & 4.00 \\
\hline $25^{\text {th }}$ percentile & & & 2.604 & 351.00 & 508.00 & 582.00 & 322.50 & 45.00 & 19.00 & 13.00 & 9.00 \\
\hline $75^{\text {th }}$ percentile & & 1.0 & 2.625 & 350.00 & 219.50 & 128.5 & 35.00 & 10.00 & 5.00 & 4.00 & 3.00 \\
\hline $50^{\text {th }}$ percentile & & & 2.661 & 350.5 & 328.00 & 247.00 & 63.00 & 14.00 & 8.00 & 5.00 & 4.00 \\
\hline $25^{\text {th }}$ percentile & & & 2.627 & 350.00 & 468.50 & 576.50 & 349.00 & 48.00 & 18.00 & 12.00 & 8.00 \\
\hline $75^{\text {th }}$ percentile & & 1.3 & 2.677 & 351.00 & 220.00 & 134.00 & 37.00 & 10.00 & 5.00 & 3.00 & 3.00 \\
\hline $50^{\text {th }}$ percentile & & & 2.735 & 350.00 & 329.00 & 270.00 & 79.00 & 16.00 & 7.00 & 5.00 & 4.00 \\
\hline $25^{\text {th }}$ percentile & & & 2.685 & 350.50 & 547.00 & 694.50 & 647.00 & 101.00 & 28.00 & 14.00 & 8.00 \\
\hline $75^{\text {th }}$ percentile & 0.9 & 0.9 & 2.250 & 350.00 & 253.00 & 156.00 & 37.00 & 13.00 & 7.00 & 5.00 & 3.00 \\
\hline $50^{\text {th }}$ percentile & & & 2.262 & 350.00 & 307.00 & 215.00 & 50.00 & 16.00 & 9.00 & 7.00 & 5.00 \\
\hline $25^{\text {th }}$ percentile & & & 2.250 & 350.00 & 405.00 & 357.00 & 103.00 & 27.00 & 16.00 & 12.00 & 10.00 \\
\hline $75^{\text {th }}$ percentile & & 1.0 & 2.324 & 349.00 & 256.00 & 149.00 & 37.00 & 12.00 & 7.00 & 5.00 & 3.00 \\
\hline $50^{\text {th }}$ percentile & & & 2.340 & 351.00 & 319.00 & 228.00 & 50.00 & 15.00 & 9.00 & 7.00 & 5.00 \\
\hline $25^{\text {th }}$ percentile & & & 2.323 & 350.50 & 412.00 & 358.00 & 112.00 & 26.00 & 15.00 & 11.00 & 9.00 \\
\hline $75^{\text {th }}$ percentile & & 1.3 & 2.491 & 350.00 & 246.00 & 157.00 & 38.00 & 11.00 & 6.00 & 4.00 & 3.00 \\
\hline $50^{\text {th }}$ percentile & & & 2.510 & 351.00 & 320.00 & 242.00 & 60.00 & 14.00 & 8.00 & 6.00 & 4.00 \\
\hline $25^{\text {th }}$ percentile & & & 2.488 & 349.00 & 423.00 & 412.00 & 176.00 & 31.00 & 14.00 & 10.00 & 7.00 \\
\hline $75^{\text {th }}$ percentile & 0.95 & 0.9 & 1.798 & 350.00 & 273.00 & 165.00 & 45.00 & 16.00 & 10.00 & 7.00 & 5.00 \\
\hline $50^{\text {th }}$ percentile & & & 1.792 & 350.00 & 306.00 & 194.00 & 52.00 & 19.00 & 12.00 & 9.00 & 7.00 \\
\hline $25^{\text {th }}$ percentile & & & 1.793 & 349.00 & 376.00 & 298.00 & 83.00 & 29.00 & 19.00 & 15.00 & 13.00 \\
\hline $75^{\text {th }}$ percentile & & 1.0 & 1.921 & 350.00 & 251.00 & 162.00 & 41.00 & 15.00 & 9.00 & 6.00 & 4.00 \\
\hline $50^{\text {th }}$ percentile & & & 1.921 & 350.00 & 311.00 & 199.00 & 48.00 & 17.00 & 11.00 & 9.00 & 7.00 \\
\hline $25^{\text {th }}$ percentile & & & 1.921 & 352.00 & 364.00 & 294.00 & 78.00 & 27.00 & 17.00 & 14.00 & 12.00 \\
\hline $75^{\text {th }}$ percentile & & 1.3 & 2.211 & 349.00 & 260.00 & 164.00 & 39.00 & 12.00 & 7.00 & 6.00 & 4.00 \\
\hline $50^{\text {th }}$ percentile & & & 2.228 & 350.5 & 326.00 & 234.00 & 51.00 & 14.00 & 9.00 & 7.00 & 6.00 \\
\hline $25^{\text {th }}$ percentile & & & 2.214 & 349.50 & 388.00 & 341.00 & 103.00 & 23.00 & 14.00 & 11.00 & 9.00 \\
\hline
\end{tabular}

Next, we compare the OOC performance of GWMA-EX chart with EWMA-EX chart and thereafter against the GWMA- $\bar{X}$ chart for the case of process parameter being unknown (Case 
U). For this purpose, we consider $\operatorname{logistic}\left(0, \frac{\sqrt{3}}{\pi}\right)$, uniform $(-\sqrt{3}, \sqrt{3})$ and $\operatorname{Laplace}\left(0, \frac{1}{\sqrt{2}}\right)$ as non-normal symmetric process distributions. The parameters are chosen for each distribution so that the mean is 0 and variance is 1 . As skewed alternatives, we consider gamma distribution with shape parameters 1,2 and 3 and scale parameter taken to be 1 without loss of generality. A summary of the findings is as follows:

(a) GWMA-EX chart vs. EWMA-EX chart and GWMA-X chart under symmetric distributions

It has been observed from Tables 1 and 2 that GWMA-EX chart, for a suitably chosen $\alpha \neq 1$, often outperforms EWMA-EX chart (i.e., when $\alpha=1$ ) under standard normal distribution. But the assumption of normality might fail in practice and in that case, it is important to see whether the parameter combination for GWMA-EX chart that yields better performance than EWMA-EX chart for normality still works well for non-normal distributions. For this purpose, we consider the combination $q=0.9, \alpha=0.7, L=1.464$ for GWMA-EX chart and the sample size $m=49, n=5$. The design parameter $q$ is taken to be the same for EWMAEX chart and thus we take $q=0.9, \alpha=1$ and $L=1.819$ for EWMA-EX chart. The idea behind taking the same design parameter for GWMA-EX and EWMA-EX charts lies in the fact that GWMA-EX chart is a generalization of EWMA-EX chart, and so it is of interest to see whether the same design parameter $q$ can provide better performance when adjustment parameter $\alpha$ is varied from 1 .

Table 5 shows that the parameter combination $q=0.9, \alpha=0.7, L=1.464$ for GWMA-EX chart that gives better performance than EWMA-EX chart for normality is also providing better performance for the considered non-normal symmetric distributions. For example, when the process follows Laplace $\left(0, \frac{1}{\sqrt{2}}\right)$ distribution and $\delta=0.1$, GWMA-EX chart with 
parameters $q=0.9, \alpha=0.7, L=1.464$ has $A R L_{1}=241.49$, while EWMA-EX chart with parameters $q=0.9, \alpha=1$ and $L=1.819$ has $A R L_{1}=252.63$.

We also compare the performance of GWMA-EX chart with GWMA- $\bar{X}$ chart for normal distribution designed under Case U. For a detail study on GWMA- $\bar{X}$ chart for normal distribution, the reader is referred to Sheu and Lin [2].

By adopting a design procedure similar to the one in Sheu and Lin [2], we construct a GWMA $\bar{X}$ chart for normal distribution for Case U, i.e., when the process location is unknown. The design parameter $q=0.9$ and the adjustment parameter $\alpha=0.7$ for GWMA$\bar{X}$ chart are taken to be the same as the competing GWMA-EX chart. The idea is to see whether the same $(q, \alpha)$ combination provides similar performance and robustness under different distributions when exceedance statistic is used instead of $\bar{X}$ in the GWMA model or there is improvement. In our study, as specified before, we take the process location 0 and variance 1, without loss of generality. The usual approach for designing parametric control charts for Case $\mathrm{U}$ is to estimate the unknown process parameters from IC Phase I sample, use those estimates to obtain the control limits, and then study the chart's run-length properties. For the chosen value of $(q=0.9, \alpha=0.7), L=3.290$ is obtained for which the attained $A R L_{0}=370.99$ for GWMA- $\bar{X}$ chart in Case $\mathrm{U}$ which is close to the nominal $A R L_{0}^{*}=370$ for $m=49, n=5$. Table 5 shows that, for normal process distribution, GWMA- $\bar{X}$ chart performs much better than GWMA-EX chart and EWMA-EX chart, but this is not surprising as the GWMA- $\bar{X}$ chart is designed under the normality assumption and is therefore expected to perform better than its nonparametric counterparts when the underlying distribution satisfies the distributional assumption for the process. However, when the process distribution departs from normality, GWMA- $\bar{X}$ chart designed for normality becomes volatile in its IC behaviour as the attained $A R L_{0}$ starts moving farther from the nominal $A R L_{0}^{*}=370$. For instance, the attained $A R L_{0}$ for GWMA- $\bar{X}$ chart is 401.32 and 289.19 when the 
underlying distributions are uniform $(-\sqrt{3}, \sqrt{3})$ and Laplace $\left(0, \frac{1}{\sqrt{2}}\right)$, respectively. On the other hand, the nonparametric counterparts such as GWMA-EX chart and EWMA-EX chart are IC robust under non-normality. According to Appendix $\mathrm{A} 1$ and $\mathrm{A} 2$, the attained $A R L_{0}$ for GWMA-EX chart and EWMA-EX chart should not differ for non-normality when the process is IC. The small variation in the obtained results in Table 5 is due to variability arising from Monte Carlo simulation. The non-robust behaviour of GWMA- $\bar{X}$ chart for nonnormality makes it to be unsuitable for use in practice when the underlying process distribution is either not known or cannot be tested.

Table 5: $A R L$ values for the GWMA-EX, EWMA-EX and GWMA- $\bar{X}$ charts for various shifts $\delta$ when $A R L_{0}^{*}=370$ and $m=49, n=5$ under symmetric distributions.

\begin{tabular}{|c|c|ccc|cccc|}
\hline$\delta$ & Chart & $q$ & $\alpha$ & $L$ & normal $(0,1)$ & $\operatorname{logistic}\left(0, \frac{\sqrt{3}}{\pi}\right)$ & uniform $(-\sqrt{3}, \sqrt{3})$ & Laplace $\left(0, \frac{1}{\sqrt{2}}\right)$ \\
\hline \multirow{3}{*}{0.00} & GWMA-EX & 0.9 & 0.7 & 1.464 & 372.82 & 369.26 & 369.26 & 371.33 \\
& EWMA-EX & 0.9 & 1.0 & 1.819 & 368.93 & 378.26 & 378.26 & 370.77 \\
& GWMA- $\bar{X}$ & 0.9 & 0.7 & 3.290 & 370.99 & 341.00 & 401.32 & 289.19 \\
\hline \multirow{3}{*}{0.05} & GWMA-EX & 0.9 & 0.7 & 1.464 & 352.92 & 343.83 & 344.27 & 318.09 \\
& EWMA-EX & 0.9 & 1.0 & 1.819 & 355.72 & 351.02 & 366.68 & 333.44 \\
& GWMA- $\bar{X}$ & 0.9 & 0.7 & 3.290 & 343.99 & 317.19 & 382.37 & 276.60 \\
\hline \multirow{3}{*}{0.10} & GWMA-EX & 0.9 & 0.7 & 1.464 & 323.44 & 310.37 & 338.19 & 241.49 \\
& EWMA-EX & 0.9 & 1.0 & 1.819 & 336.79 & 314.61 & 351.14 & 252.63 \\
& GWMA- $\bar{X}$ & 0.9 & 0.7 & 3.290 & 292.85 & 270.34 & 330.298 & 231.81 \\
\hline \multirow{3}{*}{0.25} & GWMA-EX & 0.9 & 0.7 & 1.464 & 171.05 & 133.18 & 246.76 & 58.82 \\
& EWMA-EX & 0.9 & 1.0 & 1.819 & 180.44 & 139.89 & 251.41 & 57.82 \\
& GWMA- $\bar{X}$ & 0.9 & 0.7 & 3.290 & 82.04 & 81.12 & 86.02 & 71.78 \\
\hline \multirow{3}{*}{0.50} & GWMA-EX & 0.9 & 0.7 & 1.464 & 31.70 & 21.25 & 82.72 & 12.56 \\
& EWMA-EX & 0.9 & 1.0 & 1.819 & 29.36 & 20.15 & 86.74 & 11.25 \\
& GWMA- $\bar{X}$ & 0.9 & 0.7 & 3.290 & 14.87 & 15.12 & 14.71 & 15.11 \\
\hline \multirow{3}{*}{1.00} & GWMA-EX & 0.9 & 0.7 & 1.464 & 7.68 & 6.97 & 10.75 & 6.37 \\
& EWMA-EX & 0.9 & 1.0 & 1.819 & 6.79 & 6.26 & 9.87 & 5.76 \\
& GWMA- $\bar{X}$ & 0.9 & 0.7 & 3.290 & 5.13 & 5.21 & 5.20 & 5.11 \\
\hline
\end{tabular}

(b) GWMA-EX chart vs. EWMA-EX chart and GWMA-X chart under skewed distributions

In order to study the performance of GWMA-EX chart, EWMA-EX chart and GWMA- $\bar{X}$ chart for skewed distributions, we consider $\operatorname{gamma}(k, \theta)$ distribution, where $k$ is the shape parameter and $\theta$ is the scale parameter. It should be noted that, unlike in the case of symmetric distributions considered, $\operatorname{gamma}(k, \theta)$ distribution has mean and variance as functions of parameters $k$ and $\theta$. Thus, for a given $k$, shift in scale parameter $\theta$ would effect 
change in both mean and variance simultaneously. Therefore, for $\operatorname{gamma}(k, \theta)$ distribution, it is not possible to consider mean 0 and variance 1 like we did earlier for symmetric distributions.

We denote the IC scale parameter as $\theta_{0}$ and OOC scale parameter as $\theta_{1}=\delta \theta_{0}$. Note that we define the shift $\delta=\frac{\theta_{1}}{\theta_{0}}$ differently for the gamma distribution than for symmetric distributions. The reason can be explained as follows: If $X \sim \operatorname{gamma}(k, \theta)$, then $Y=\frac{X}{\theta} \sim \operatorname{gamma}(k, 1)$. Therefore, the IC scale parameter $\theta_{0}$ can be taken as 1 without loss of generality. We define $\frac{\theta_{1}}{\theta_{0}}=\delta$ and thus, taking $\theta_{0}=1$ without loss of generality, we have $\theta_{1}=\delta$. For a shift $\delta$, it is easy to see that, $\frac{Y}{\delta} \sim$ gamma $\left(k, \frac{1}{\delta}\right)$ and thus, irrespective of the true value of $\theta_{0}, \theta_{1}, \frac{X}{\theta_{1}}=\frac{Y}{\delta}$ would have the same distribution as long as the ratio $\delta$ is the same. Thus, the chart properties will be the same for different $\theta_{0}$ and $\theta_{1}$ as long as their ratio $\delta$ is the same. But, the absolute difference does not take this into consideration since, for the absolute difference $\left|\theta_{1}-\theta_{0}\right|$, the effect of shift depends on the magnitude of $\theta_{0}$ itself. For instance, 0.05 shift in 1 is not the same as 0.05 shift in 5 . So, the chart useful for shift 0.05 in $\theta_{0}=1$ cannot be used for shift 0.05 in $\theta_{0}=5$ if the chart is designed for absolute difference. Taking $\theta_{0}=1$ without loss of generality would make the chart useful for any IC $\theta_{0}$, but the OOC performance would vary for different $\theta_{0}$ and $\theta_{1}$ even if $\left|\theta_{1}-\theta_{0}\right|$ is the same. On the other hand, shift in ratio of 0.05 can be used for any $\theta_{0}, \theta_{1}$ as long as their ratio is 0.05 . We now consider $\delta=0.975,0.95$, $0.9,0.8,0.7$ for the OOC process and $\delta=1$ for the IC process. The construction of GWMAEX chart for gamma $(k, \theta)$ distribution is same as for normal or other symmetric distributions. But, for GWMA- $\bar{X}$ chart in Case $\mathrm{U}$, the control limits used for normal distribution are clearly unsuitable for $\operatorname{gamma}(k, \theta)$ distribution. This is due to the fact that the distribution mean and variance are no longer 0 and 1 , respectively. If we get the estimate of the process mean from IC Phase I sample and plug that in to the control limits with a blunt choice of variance 1, the 
starting value being replaced by the estimated process mean, GWMA- $\bar{X}$ chart will perform nonsensically when the process is IC. For example, GWMA- $\bar{X}$ chart with $(q=0.9, \alpha=$ 0.7, $L=3.290)$ has $A R L_{0}=341.85$ for gamma(1,1) distribution, $A R L_{0}=59.99$ for gamma(2,1) distribution, and $A R L_{0}=30.66$ for $\operatorname{gamma}(3,1)$ distribution in Case $\mathrm{U}$ when $m=49, n=5$. It is thus clear that the usual GWMA- $\bar{X}$ chart designed under normal assumption does not work well for skewed distributions.

Note that the control limits for GWMA- $\bar{X}$ chart are of the form $\left(\mu \pm L \frac{\sigma}{\sqrt{n}}\right)$ and since the assumption of variance 1 is not reasonable, we take the estimate of both process mean $\mu$ and standard deviation $\sigma$ from the IC Phase I sample and plug these estimates, denoted by $\hat{\mu}$ and $\hat{\sigma}$, into the control limits. The starting value is also taken as $k \hat{\theta}$ instead of 0 . Table 6 shows that GWMA- $\bar{X}$ chart is far from IC robustness and it often encounters bias in run-length distribution in the case of skewed process distribution. For example, GWMA- $\bar{X}$ chart with $(q=0.9, \alpha=0.7, L=3.290)$ and $m=49, n=5$ has $A R L_{0}=408.82$ for $\operatorname{gamma}(1,1)$ distribution, $A R L_{0}=405.98$ for $\operatorname{gamma}(2,1)$ distribution, and $A R L_{0}=413.81$ for gamma $(3,1)$ when the process standard deviation is also replaced by its estimate. Table 6 shows that, for gamma distribution with shape parameters $k=1,2,3$, GWMA-EX chart outperforms EWMA-EX chart for all shift $\delta \geq 0.7$ except for the case of $k=3$ and $\delta=0.7$ where EWMA-EX chart occasionally has earlier detection than GWMA-EX chart. 
Table 6: $A R L$ values for the GWMA-EX, EWMA-EX and GWMA- $\bar{X}$ charts for various shifts $\delta$ when $A R L_{0}^{*}=370$ and $m=49, n=5$ under skewed distributions.

\begin{tabular}{|c|c|ccc|c|c|c|}
\hline$\delta$ & Chart & $q$ & $\alpha$ & $L$ & gamma(1,1) & gamma(2,1) & gamma(3,1) \\
\hline \multirow{3}{*}{1.00} & GWMA-EX & 0.9 & 0.7 & 1.464 & 368.44 & 373.83 & 372.14 \\
& EWMA-EX & 0.9 & 1.0 & 1.819 & 371.33 & 365.10 & 371.79 \\
& GWMA- $\bar{X}$ & 0.9 & 0.7 & 3.290 & 408.82 & 405.98 & 413.81 \\
\hline \multirow{3}{*}{0.975} & GWMA-EX & 0.9 & 0.7 & 1.464 & 363.17 & 369.06 & 366.26 \\
& EWMA-EX & 0.9 & 1.0 & 1.819 & 367.73 & 375.94 & 356.70 \\
& GWMA- $\bar{X}$ & 0.9 & 0.7 & 3.290 & 473.63 & 495.82 & 504.24 \\
\hline \multirow{3}{*}{0.95} & GWMA-EX & 0.9 & 0.7 & 1.464 & 359.01 & 333.14 & 333.87 \\
& EWMA-EX & 0.9 & 1.0 & 1.819 & 363.28 & 343.60 & 337.33 \\
& GWMA- $\bar{X}$ & 0.9 & 0.7 & 3.290 & 571.83 & 612.79 & 555.01 \\
\hline \multirow{3}{*}{0.9} & GWMA-EX & 0.9 & 0.7 & 1.464 & 324.08 & 274.66 & 256.92 \\
& EWMA-EX & 0.9 & 1.0 & 1.819 & 325.94 & 289.15 & 250.09 \\
& GWMA- $\bar{X}$ & 0.9 & 0.7 & 3.290 & 747.99 & 662.64 & 546.23 \\
\hline \multirow{3}{*}{0.7} & GWMA-EX & 0.9 & 0.7 & 1.464 & 226.12 & 134.39 & 75.63 \\
& EWMA-EX & 0.9 & 1.0 & 1.819 & 231.50 & 137.93 & 82.49 \\
& GWMA- $\bar{X}$ & 0.9 & 0.7 & 3.290 & 828.29 & 368.39 & 159.18 \\
\hline & GWMA-EX & 0.9 & 0.7 & 1.464 & 117.05 & 39.19 & 19.68 \\
& EWMA-EX & 0.9 & 1.0 & 1.819 & 121.28 & 41.24 & 18.58 \\
& GWMA- $\bar{X}$ & 0.9 & 0.7 & 3.290 & 445.61 & 63.04 & 18.01 \\
\hline
\end{tabular}

As a general recommendation for implementation of the GWMA-EX chart, it can be said that the value of $q$ in $0.8 \leq q \leq 0.95$ and $\alpha$ in the interval 0.7 to 0.9 should be useful in detecting a shift of $\delta<0.5$ while $\alpha>1$ should be useful in detecting larger shifts. An example is provided in the following section to illustrate the proposed GWMA-EX chart.

\section{Illustrative example}

To illustrate the application of the proposed GWMA-EX chart, we draw 49 samples of size $n=5$ from normal $(0,1)$ distribution as Phase I dataset to estimate the process median. The median of Phase I sample is 0.165 . We also draw 200 Phase II random samples from normal $(0.25,1)$ distribution which can be regarded as OOC observations from a process with location shift $\delta=0.25$. Table 1 shows that, for $\delta=0.25$, GWMA-EX chart with $(q=0.9$, $\alpha=0.7, L=1.464)$ has $A R L_{1}=171.05$ and EWMA-EX chart with $(q=0.9, \alpha=1.0, L=$ 1.819) has $A R L_{1}=180.44$. We take $q=0.9, \alpha=0.7, L=1.464$ and following the same approach as in Section 3, GWMA-EX chart is designed. Control limits for GWMA-EX chart are obtained as $U C L=3.077$ and $L C L=1.923$. An EWMA-EX chart is also designed with $q=0.9, \alpha=1.0, L=1.819$ and the control limits are $U C L=3.287$ and $L C L=1.713$. The 
Figure 4. GWMA-EX and EWMA-EX chart implemented on simulated data.

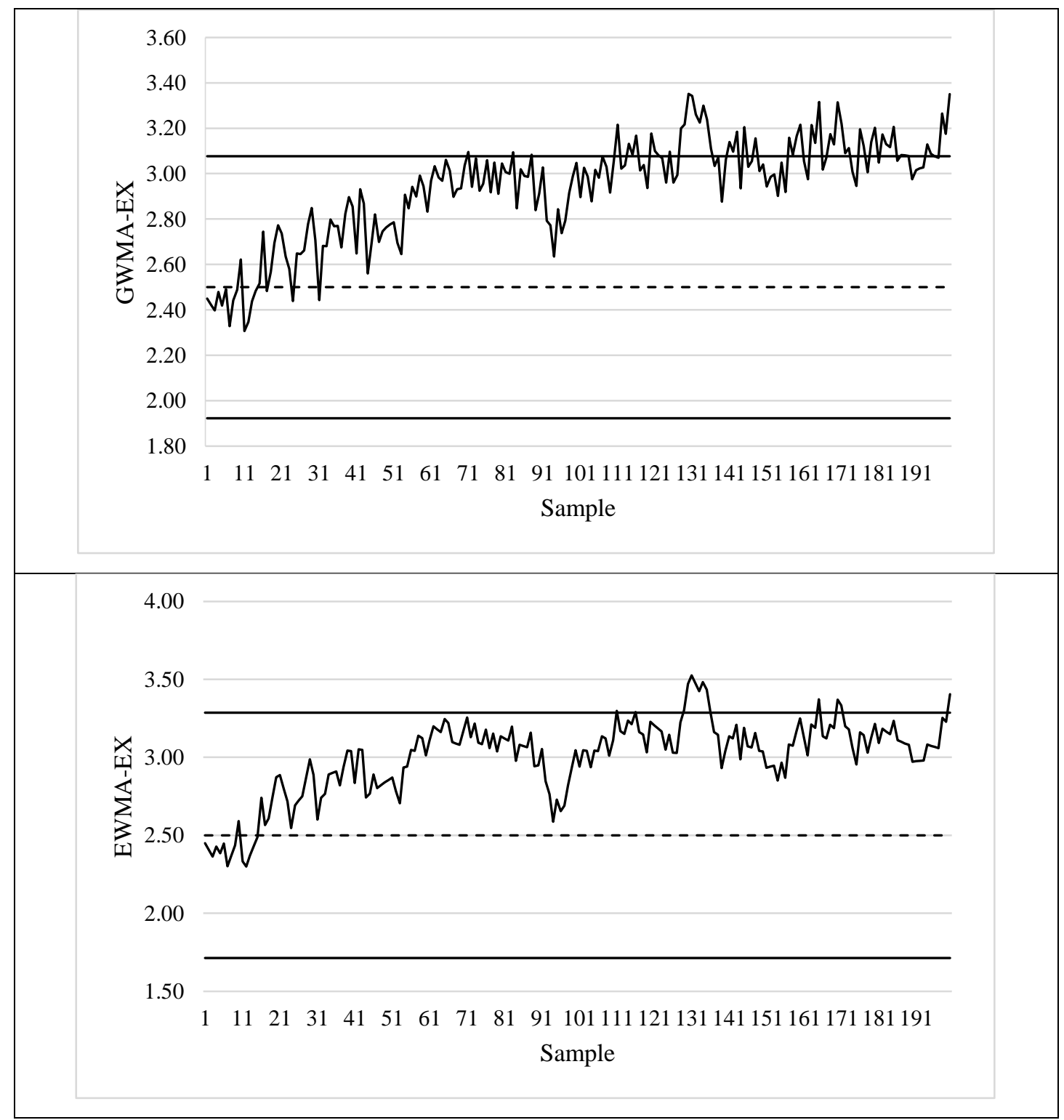

attained $A R L_{0}$ for these two charts are close to 370 which put them at a similar IC performance level and are therefore comparable. As described before, the chart parameters $(q, \alpha, L)$ must be chosen in a way so that the attained $A R L_{0}$ is close to the nominal $A R L_{0}^{*}$ when designing a GWMA-EX chart for monitoring a streaming process. The two control charts are displayed in Fig. 4. From these, we observe the following:

- GWMA-EX chart detects shift $\delta=0.25$ much faster than EWMA-EX chart which is evident from the average detection ability of these two charts in Table 1; 
- $\quad$ Both of the charts are using steady-state control limits. The centreline is equal to the unconditional IC expectation of the plotting statistic;

- The vertical axis is not at the original scale of measurement because the building block for GWMA-EX chart and EWMA-EX chart is the exceedance statistic.

\section{Concluding remarks}

The rigid assumption of normality for the process distribution may not hold in practice while designing a control chart for monitoring a streaming process. The performance of the classical GWMA- $\bar{X}$ chart becomes worse under skewed distributions when the process distribution is unknown. A distribution-free GWMA control chart based on exceedance statistic, referred to as GWMA-EX chart, has been constructed in this paper for a process for which information on the underlying process distribution as well as the process median are not available. Design and relative performance study of the proposed GWMA-EX chart has been carried out. It has been observed that the proposed chart, with no information on the true IC process median or process distribution, is robust to non-normality when the process is IC and it performs just as well and in many cases better than the existing EWMA chart based on exceedance statistic when the shift is small.

\section{Acknowledgement}

The financial assistance of the National Research Foundation (NRF) towards this research is hereby acknowledged. Opinion expressed and conclusion arrived at are those of the authors and are not necessarily to be attributed to the NRF. Part of this research has been funded by STATOMET, Department of Statistics, University of Pretoria, South Africa.

\section{Declaration of Interest}

No potential conflict of interest was reported by the authors. 


\section{Reference}

[1] Montgomery, D. C. (2009). Statistical Quality Control: A Modern Introduction, 6th ed. John Wiley \& Sons: Hoboken, New Jersey.

[2] Sheu, S. H. \& Lin, T. C. (2003). The generally weighted moving average control chart for detecting small shifts in the process mean. Quality Engineering, 16(2), 209-231.

[3] Hsu, B. M., Lai, P. J., Shu, M. H. \& Hung, Y. Y. (2009). A comparative study of the monitoring performance for weighted control charts. Journal of Statistics and Management Systems, 12(2), 207-228.

[4] Chakraborty, N., Human, S. W. \& Balakrishnan, N. (2017). A generally weighted moving average chart for time between events. Communications in Statistics-Simulation and Computation, DOI: http://dx.doi.org/10.1080/03610918.2016.1252397.

[5] Chakraborty, N., Chakraborti, S., Human, S. W. \& Balakrishnan, N. (2016). A Generally Weighted Moving Average Signed-rank Control Chart. Quality and Reliability Engineering International, 32(8), 2835-2845.

[6] Lu, S. L. (2015). An extended nonparametric exponentially weighted moving average sign control chart. Quality and Reliability Engineering International, 31(1), 3-13.

[7] Balakrishnan, N. \& Ng, H. T. (2006). Precedence-Type Tests and Applications. John Wiley \& Sons: Hoboken, New Jersey.

[8] Chakraborti, S., Laan, P. \& Wiel, M. A. (2004). A class of distribution-free control charts. Journal of the Royal Statistical Society: Series C, 53(3), 443-462.

[9] Graham, M. A., Mukherjee, A. \& Chakraborti, S. (2012). Distribution-free exponentially weighted moving average control charts for monitoring unknown location. Computational Statistics \& Data Analysis, 56(8), 2539-2561. 
[10] Graham, M. A., Chakraborti, S. \& Mukherjee, A. (2014). Design and implementation of CUSUM exceedance control charts for unknown location. International Journal of Production Research, 52(18), 5546-5564.

[11] Mukherjee, A., Graham, M. A. \& Chakraborti, S. (2013). Distribution-free exceedance CUSUM control charts for location. Communications in Statistics-Simulation and Computation, 42(5), 1153-1187.

[12] Nakagawa, T. \& Osaki, S. (1975). The discrete Weibull distribution. IEEE Transactions on Reliability, 24(5), 300-301.

[13] Sheu, S. H. \& Yang, L. (2006). The generally weighted moving average control chart for monitoring the process median. Quality engineering, 18(3), 333-344.

\section{Appendix}

A number of results pertaining the distribution of the exceedance statistic are presented here.

A1. Conditional on $X_{(r)}$, the exceedance statistics $V_{i}, i=1,2, \ldots$, are independent and identically distributed as binomial with parameters $\left(n, p_{r}\right)$, where $n$ is the sample size and $p_{r}=1-G\left(x_{r} \mid X_{(r)}=x_{r}\right)$, with $G($.$) being the c.d.f. of the test sample \left(Y_{i 1}, Y_{i 2}, \ldots, Y_{i n}\right)$.

Proof: Given $X_{(r)}$, the event that every $Y$ observation in a test sample is either smaller or larger than $X_{(r)}$ follows the properties of a Bernoulli trial. Note that the number of $Y$ observations smaller or larger than $X_{(r)}$, given $X_{(r)}$, are independent for every Phase II sample. Thus, the random variable $V_{i}$ denoting the number of exceedances given by the number of $Y$ observations in the $i^{\text {th }}$ test sample that exceed $X_{(r)}$ is binomially distributed with parameters $\left(n, p_{r}\right)$, given $X_{(r)}$, where the probability of success is $p_{r}=\operatorname{Pr}[Y>$ $\left.X_{(r)} \mid X_{(r)}=x_{r}\right]=1-G\left(x_{r} \mid X_{(r)}=x_{r}\right)$. 
A2. The unconditional IC distribution of $V_{i}$, for all $i=1,2, \ldots$, is distribution-free and is given by the p.m.f. $\operatorname{Pr}\left(V_{i}=v\right)=\frac{\left(\begin{array}{c}v+m-r \\ v\end{array}\right)\left(\begin{array}{c}n-v+r-1 \\ n-v\end{array}\right)}{\left(\begin{array}{c}m+n \\ n\end{array}\right)}, v=0,1,2, \ldots, n$.

Proof: From A1, $\operatorname{Pr}\left[V_{i}=v \mid X_{(r)}=x_{r}\right]$

$=\left(\begin{array}{l}n \\ v\end{array}\right) p_{r}^{v}\left(1-p_{r}\right)^{n-v}=\left(\begin{array}{l}n \\ v\end{array}\right)\left(1-G\left(x_{r}\right)\right)^{v} G\left(x_{r}\right)^{n-v}, v=0,1,2, \ldots, n$.

Therefore, by unconditioning, we obtain

$$
\begin{aligned}
& \operatorname{Pr}\left[V_{i}=v\right]=E_{X_{(r)}}\left(\operatorname{Pr}\left[V_{i}=v \mid X_{(r)}=x_{r}\right]\right) \\
& =\int_{-\infty}^{\infty}\left(\begin{array}{l}
n \\
v
\end{array}\right)\left(1-G\left(x_{r}\right)\right)^{v} G\left(x_{r}\right)^{n-v} \frac{m !}{(r-1) !(m-r) !} F\left(x_{r}\right)^{r-1}\left(1-F\left(x_{r}\right)\right)^{m-r} f\left(x_{r}\right) d x_{r} .
\end{aligned}
$$

For IC process, we have $G=F$. Thus, the IC unconditional distribution of $V_{i}$ is given by

$$
\begin{aligned}
& \operatorname{Pr}\left[V_{i}=v\right]=\left(\begin{array}{l}
n \\
v
\end{array}\right) \frac{m !}{(r-1) !(m-r) !} \int_{-\infty}^{\infty} F\left(x_{r}\right)^{n-v+r-1}\left(1-F\left(x_{r}\right)\right)^{m+v-r} f\left(x_{r}\right) d x_{r} \\
& =\frac{n !}{v !(n-v) !} \frac{m !}{(r-1) !(m-r) !} \frac{(n-v+r-1) !(m+v-r) !}{(m+n) !} \\
& =\frac{\left(\begin{array}{c}
n-v+r-1 \\
n-v
\end{array}\right)\left(\begin{array}{c}
m+v-r \\
v
\end{array}\right)}{\left(\begin{array}{c}
m+n \\
n
\end{array}\right)} \text { for } v=0,1,2, \ldots, n, \text { as required. }
\end{aligned}
$$

A3. The unconditional IC joint distribution of $t$ number of exceedance statistics $\left(V_{1}, V_{2}, \ldots V_{t}\right)$ is distribution-free and is given by the joint p.m.f.

$\operatorname{Pr}\left[V_{1}=v_{1}, V_{2}=v_{2}, \ldots, V_{t}=v_{t}\right]=\frac{\prod_{k=1}^{t}\left(\begin{array}{c}n k-\sum_{i=1}^{k} v_{i}+r-1 \\ n-v_{k}\end{array}\right) \prod_{k=1}^{t}\left(\begin{array}{c}m-r+\sum_{i=1}^{k} v_{i} \\ v_{k}\end{array}\right)}{\prod_{k=1}^{t}\left(\begin{array}{c}m+n k \\ n\end{array}\right)}$

where $v_{i}=0,1,2, \ldots, n$ for all $i=1,2, \ldots, t$.

Proof: The conditional IC joint distribution of the random variables $\left(V_{1}, V_{2}, \ldots, V_{t}\right)$, when $F=G$, is given by 
$\operatorname{Pr}\left[V_{1}=v_{1}, V_{2}=v_{2}, \ldots, V_{t}=v_{t} \mid X_{(r)}=x_{r}\right]=\prod_{i=1}^{t}\left(\begin{array}{c}n \\ v_{i}\end{array}\right)\left(1-F\left(x_{r}\right)\right)^{v_{i}} F\left(x_{r}\right)^{n-v_{i}}$

for $v_{i}=0,1,2, \ldots, n$ for all $i=1,2, \ldots, t$. By unconditioning over $X_{(r)}$,

$\operatorname{Pr}\left[V_{1}=v_{1}, V_{2}=v_{2}, \ldots, V_{t}=v_{t}\right]$

$=E_{X_{(r)}}\left(\operatorname{Pr}\left[V_{1}=v_{1}, V_{2}=v_{2}, \ldots, V_{t}=v_{t} \mid X_{(r)}\right]\right)$

$=\left(\prod_{i=1}^{t}\left(\begin{array}{l}n \\ v_{i}\end{array}\right)\right) \int_{-\infty}^{\infty}\left(1-F\left(x_{r}\right)\right)^{\sum_{i=1}^{t} v_{i}} F\left(x_{r}\right)^{n t-\sum_{i=1}^{t} v_{i}} \frac{m !}{(r-1) !(m-r) !} F\left(x_{r}\right)^{r-1}\left(1-F\left(x_{r}\right)\right)^{m-r} f\left(x_{r}\right) d x_{r}$

$=\left(\prod_{i=1}^{t}\left(\begin{array}{c}n \\ v_{i}\end{array}\right)\right) \frac{m !}{(r-1) !(m-r) !} \cdot \frac{\left(m+\sum v_{i}-r\right) !\left(n t-\sum v_{i}+r-1\right) !}{(m+n t) !}$

$=\frac{\prod_{k=1}^{t}\left(\begin{array}{c}n k-\sum_{i=1}^{k} v_{i}+r-1 \\ n-v_{k}\end{array}\right) \prod_{k=1}^{t}\left(\begin{array}{c}m-r+\sum_{i=1}^{k} v_{i} \\ v_{k}\end{array}\right)}{\prod_{k=1}^{t}\left(\begin{array}{c}m+n k \\ n\end{array}\right)}$ for $v_{i}=0,1,2, \ldots, n$ for all $i=1,2, \ldots, t$,

as required.

A4. The unconditional IC expectation of $V_{i r}$ is given by $E\left(V_{i}\right)=n\left(1-\left(\frac{r}{m+1}\right)\right)$.

Proof: We have

$E\left(V_{i}\right)=E_{X_{(r)}}\left(E\left(V_{i} \mid X_{(r)}\right)\right)=E_{X_{(r)}}\left[\sum_{v=0}^{n} v \operatorname{Pr}\left[V_{i}=v \mid X_{(r)}\right]\right]$

For IC process, $F=G$, and so

$E\left(V_{i}\right)=E_{X_{(r)}}\left[\sum_{v=0}^{n} v\left(\begin{array}{l}n \\ v\end{array}\right)\left(1-F\left(x_{r}\right)\right)^{v} F\left(x_{r}\right)^{n-v}\right]=n E_{X_{(r)}}\left(1-F\left(X_{(r)}\right)\right)$.

Now,

$$
\begin{aligned}
& E_{X_{(r)}}\left(1-F\left(X_{(r)}\right)\right)=\int_{-\infty}^{\infty}\left(1-F\left(x_{r}\right)\right) \frac{m !}{(r-1) !(m-r) !} F\left(x_{r}\right)^{r-1}\left(1-F\left(x_{r}\right)\right)^{m-r} f\left(x_{r}\right) d x_{r} \\
& =\frac{m !}{(r-1) !(m-r) !} \int_{-\infty}^{\infty} F\left(x_{r}\right)^{r-1}\left(1-F\left(x_{r}\right)\right)^{m-r+1} f\left(x_{r}\right) d x_{r}
\end{aligned}
$$


$=\frac{m-r+1}{m+1}$.

Thus, the unconditional IC expectation of $V_{i}$ is given by

$E\left(V_{i}\right)=n E_{X_{(r)}}\left(1-F\left(x_{r}\right)\right)=n\left(1-\left(\frac{r}{m+1}\right)\right)$. This completes the proof.

A5. The unconditional IC variance of $V_{i}$ is given by $\operatorname{Var}\left(V_{i}\right)=\frac{n r(m-r+1)(m+n+1)}{(m+1)^{2}(m+2)}$.

Proof: For IC process, we have $F=G$. The unconditional IC variance of $V_{i}$ is, therefore,

$$
\operatorname{Var}\left(V_{i}\right)=\operatorname{Var}_{X_{(r)}}\left(E\left(V_{i} \mid X_{(r)}\right)\right)+E_{X_{(r)}}\left(\operatorname{Var}\left(V_{i} \mid X_{(r)}\right)\right)
$$

Conditional on $X_{(r)}$, the exceedance statistic $V_{i r} \sim \operatorname{Bin}\left(n, p_{r}\right)$. So, the conditional expectation and variance of $V_{i r}$ are given by, respectively,

$E\left(V_{i} \mid X_{(r)}\right)=n\left(1-F\left(x_{r}\right)\right)$ and $\operatorname{Var}\left(V_{i} \mid X_{(r)}\right)=n F\left(x_{r}\right)\left(1-F\left(x_{r}\right)\right)$.

Therefore, $\operatorname{Var}_{X_{(r)}}\left(E\left(V_{i} \mid X_{(r)}\right)\right)=n^{2} \operatorname{Var}_{X_{(r)}}\left(1-F\left(X_{(r)}\right)\right)$.

Now, we need to obtain $\operatorname{Var}_{X_{(r)}}\left(1-F\left(X_{(r)}\right)\right)=E_{X_{(r)}}\left(1-F\left(X_{(r)}\right)\right)^{2}-E_{X_{(r)}}^{2}\left(1-F\left(X_{(r)}\right)\right)$.

We first obtain $E_{X_{(r)}}\left(1-F\left(X_{(r)}\right)\right)^{2}$ as

$$
\begin{aligned}
& E_{X_{(r)}}\left(1-F\left(X_{(r)}\right)\right)^{2}=\int_{-\infty}^{\infty}\left(1-F\left(x_{r}\right)\right)^{2} \frac{m !}{(r-1) !(m-r) !} F\left(x_{r}\right)^{r-1}\left(1-F\left(x_{r}\right)\right)^{m-r} f\left(x_{r}\right) d x_{r} \\
& =\frac{(m-r+2)(m-r+1)}{(m+2)(m+1)}
\end{aligned}
$$

From A4, we have $E_{X_{(r)}}\left(1-F\left(X_{(r)}\right)\right)=\frac{m-r+1}{m+1}$.

So, $\operatorname{Var}_{X_{(r)}}\left(1-F\left(X_{(r)}\right)\right)=E_{X_{(r)}}\left(1-F\left(X_{(r)}\right)\right)^{2}-E_{X_{(r)}}^{2}\left(1-F\left(X_{(r)}\right)\right)$ 
$=\frac{(m-r+2)(m-r+1)}{(m+2)(m+1)}-\left(\frac{m-r+1}{m+1}\right)^{2}=\frac{r(m-r+1)}{(m+1)^{2}(m+2)}$.

Therefore, $\operatorname{Var}_{X_{(r)}}\left(E\left(V_{i} \mid X_{(r)}\right)\right)=n^{2} \operatorname{Var}_{X_{(r)}}\left(1-F\left(X_{(r)}\right)\right)=\frac{n^{2} r(m-r+1)}{(m+1)^{2}(m+2)}$.

Now, we need to calculate

$E_{X_{(r)}}\left(\operatorname{Var}\left(V_{i} \mid X_{(r)}\right)\right)=n E_{X_{(r)}}\left(F\left(X_{(r)}\right)\left(1-F\left(X_{(r)}\right)\right)\right)$

for which we have

$$
\begin{aligned}
& E_{X_{(r)}}\left(F\left(X_{(r)}\right)\left(1-F\left(X_{(r)}\right)\right)\right) \\
& =\int_{-\infty}^{\infty} F\left(x_{r}\right)\left(1-F\left(x_{r}\right)\right) \frac{m !}{(r-1) !(m-r) !} F\left(x_{r}\right)^{r-1}\left(1-F\left(x_{r}\right)\right)^{m-r} f\left(x_{r}\right) d x_{r} \\
& =\frac{r(m-r+1)}{(m+2)(m+1)} .
\end{aligned}
$$

Thus,

$$
E_{X_{(r)}}\left(\operatorname{Var}\left(V_{i} \mid X_{(r)}\right)\right)=n E_{X_{(r)}}\left(F\left(X_{(r)}\right)\left(1-F\left(X_{(r)}\right)\right)\right)=\frac{n r(m-r+1)}{(m+2)(m+1)}
$$

By substituting (ii) and (iii) in (i), we get

$\operatorname{Var}\left(V_{i}\right)=\operatorname{Var}_{X_{(r)}}\left(E\left(V_{i} \mid X_{(r)}\right)\right)+E_{X_{(r)}}\left(\operatorname{Var}\left(V_{i} \mid X_{(r)}\right)\right)=\frac{n r(m-r+1)(m+n+1)}{(m+1)^{2}(m+2)}$ as required.

A6. $A R L \mid X_{(r)}=\sum_{r=0}^{\infty} I_{\mathrm{r}}$ where $I_{r}=\operatorname{Pr}\left[\cap_{i=1}^{r} A_{i}^{c} \mid X_{(r)}\right]=\sum_{L_{1}}^{U_{1}} \sum_{L_{2}}^{U_{2}} \ldots \sum_{L_{r}}^{U_{r}}\left(\prod_{i=1}^{r} \operatorname{Pr}\left[V_{i}=\right.\right.$ $\left.\left.v_{i} \mid X_{(r)}\right]\right)$ for $r=1,2,3, \ldots$ and $I_{0}=1$.

Proof: The conditional run-length probabilities can be written as, $\operatorname{Pr}\left[R=r \mid X_{(r)}\right]=$ $\operatorname{Pr}\left[\cap_{i=1}^{r-1} A_{i}^{c} \mid X_{(r)}\right]-\operatorname{Pr}\left[\cap_{i=1}^{r} A_{i}^{c} \mid X_{(r)}\right]=I_{r-1}-I_{r} \quad, \quad$ for $\quad r=1, \quad 2, \quad 3, \ldots, \quad$ where $I_{r}=\operatorname{Pr}\left[\cap_{i=1}^{r} A_{i}^{c} \mid X_{(r)}\right]=\sum_{L_{1}}^{U_{1}} \sum_{L_{2}}^{U_{2}} \ldots \sum_{L_{r}}^{U_{r}}\left(\prod_{i=1}^{r} \operatorname{Pr}\left[V_{i}=v_{i} \mid X_{(r)}\right]\right)$ and $I_{0}=1 . \operatorname{Pr}\left[V_{i}=\right.$ 
$\left.v_{i} \mid X_{(r)}\right]$ is given in Appendix A1. Thus, we have $A R L \mid X_{(r)}=\sum_{r=1}^{\infty} r \operatorname{Pr}\left[R=r \mid X_{(r)}\right]$. Upon expanding and re-arranging some of the terms, we obtain

$A R L \mid X_{(r)}=\operatorname{Pr}\left[R=1 \mid X_{(r)}\right]+\sum_{r=2}^{\infty} r \operatorname{Pr}\left[R=r \mid X_{(r)}\right]=1-\operatorname{Pr}\left[A_{1}^{c} \mid X_{(r)}\right]+\sum_{r=2}^{\infty} r\left(I_{\mathrm{r}-1}-\right.$ $\left.I_{\mathrm{r}}\right)=1-\operatorname{Pr}\left[A_{1}^{c} \mid X_{(r)}\right]+I_{1}+\sum_{r=1}^{\infty} I_{\mathrm{r}}=1+\sum_{r=1}^{\infty} I_{\mathrm{r}}$, as required. 